\title{
An Overview of the Adaptive Robust DFT
}

\author{
Alexey A. Roenko, ${ }^{1}$ Vladimir V. Lukin, ${ }^{1}$ and Igor Djurovic ${ }^{2}$ \\ ${ }^{1}$ Department of Transmitters, Receivers, and Signal Processing, National Aerospace University, Kharkov, \\ 17 Chkalova St., 61070 Kharkov, Ukraine \\ ${ }^{2}$ Electrical Engineering Department, University of Montenegro, Cetinjski put bb, 81000 Podgorica, Montenegro
}

Correspondence should be addressed to Vladimir V. Lukin, vladimlukin@yahoo.com

Received 24 January 2010; Accepted 17 May 2010

Academic Editor: Ljubiša Stanković

Copyright ( $) 2010$ Alexey A. Roenko et al. This is an open access article distributed under the Creative Commons Attribution License, which permits unrestricted use, distribution, and reproduction in any medium, provided the original work is properly cited.

This paper overviews basic principles and applications of the robust DFT (RDFT) approach, which is used for robust processing of frequency-modulated (FM) signals embedded in non-Gaussian heavy-tailed noise. In particular, we concentrate on the spectral analysis and filtering of signals corrupted by impulsive distortions using adaptive and nonadaptive robust estimators. Several adaptive estimators of location parameter are considered, and it is shown that their application is preferable with respect to nonadaptive counterparts. This fact is demonstrated by efficiency comparison of adaptive and nonadaptive RDFT methods for different noise environments.

\section{Introduction}

Using Gaussian distributions, noise and other phenomena have been described over time in various applications such as communications, radars, sonars, and acoustics [1]. However, recent studies have shown that heavier-tailed probability density functions (PDFs) provide a more adequate description of noise in many practical environments [24]. In particular, Spaulding and Middleton [2] initiated this development of various complex noise models. For example, variants of contaminated [5], generalized Gaussian distributions [6] and a family of symmetric $\alpha$-stable $(\mathrm{S} \alpha \mathrm{S})$ PDFs $[3,6]$ are models widely used to describe nonGaussian noise environments. These realistic noise models have stimulated the development of robust estimators for nonlinear signal and image filtering [3, 5-7].

Theoretical developments behind linear filters have been concurrently performed in both temporal and spectral (Fourier transform) domains over the years [8]. Meanwhile, the theory of nonlinear filtering mainly focused on signal processing in the temporal domain. Typical robust filters include median, L-filters, and $\alpha$-trimmed mean filters [7] while recent contributions include more sophisticated filters such as FIR-hybrid median, weighted median, and some other nonlinear filters [7]. Although classification and terminology used for linear filters can be hardly applied to nonlinear filters [9], they are generally regarded as low-pass filters and cannot be used as pass-band, stop-band, and/or high-pass filters. Arce and his coworkers recently introduced a class of weighted median and myriad filters admitting negative weights [9-11]. These filters can be used to design nonlinear filters of all types (low-pass, high-pass, stop-band, and band-pass), while simultaneously removing impulsive noise. This development has renewed interests for the design and analysis of robust filters [12].

Practically at the same time, an alternative way to cope with non-Gaussian noise has been proposed by Katkovnik [13]. His contribution was followed by several other papers [14-17] where various robust periodogram and DFT forms were proposed. In particular, these so-called robust DFT (RDFT) methods estimate real (RE) and imaginary (IM) components of signal Fourier spectrum in a robust manner. The standard set of the robust estimators can be used for this task including M-, L-, and R-estimators [5]. In addition, the adaptive RDFT forms have been proposed as well [1420]. These transforms are further generalized in various applications including the time-frequency analysis [21, 22], radars $[23,24]$, filtering of frequency-modulated (FM) and pulse-like signals $[20,25]$, and the estimation of signal parameters $[21,26]$. 


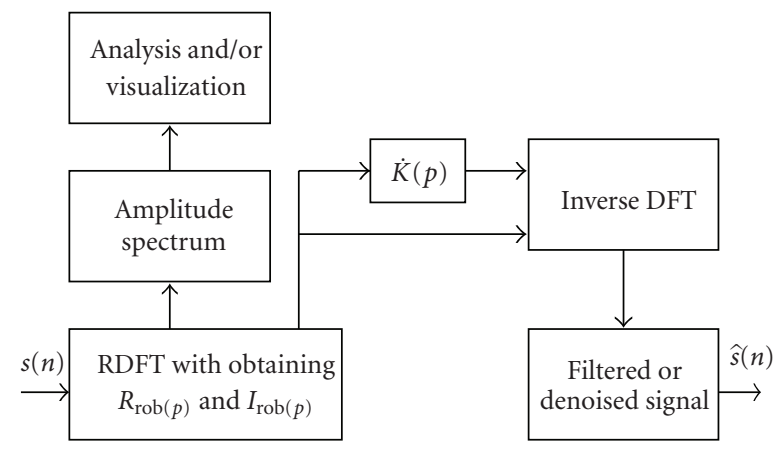

FIGURE 1: Block diagram for signal processing approach based on RDFT.

The goal of this paper is to analyze and recommend proper RDFT forms for various signal-noise scenarios. Therefore, the paper is organized as follows: we discuss design challenges in Section 2 while various adaptive and non-adaptive robust estimators are described in Section 3. The results of a numerical analysis of non-adaptive and adaptive RDFT methods are covered in Section 4.

\section{RDFT Framework and Requirements to Robust Estimators}

Consider a one-dimensional (1D) discrete signal $s(n)$ corrupted by non-Gaussian noise $v(n)$

$$
x(n)=s(n)+v(n)
$$

where $n \in[1, N]$. To filter $x(n)$ using the RDFT-based approach we implement the following steps $[13,14,16-20]$ :

(1) calculate the RDFT for the analyzed signal;

(2) multiply the obtained spectrum estimate, $\hat{X}_{\text {rob }}(p)$ ( $p \in[0, N-1]$ is the frequency index), with specified frequency characteristic $K(p)$;

(3) calculate the standard inverse DFT.

The block diagram of the RDFT-based signal processing approach is shown in Figure 1. The RDFT can be used in all applications where the standard DFT is commonly applied. For example, the RDFT is used for the time-frequency analysis in [27].

The complex-valued spectrum obtained by RDFT method can be written as

$$
\hat{X}_{\text {rob }}(p)=R_{\text {rob }}(p)+j I_{\text {rob }}(p)
$$

where $R_{\text {rob }}(p)$ and $I_{\text {rob }}(p)$ are the robust estimates of RE and IM components of the DFT. The index $p$ corresponds to frequencies $f_{p}$ as $f_{p}=p \Delta f, \Delta f=1 / N T_{S} ; R_{\text {rob }}(p)=$ $R_{\text {rob }}\left(f_{p}\right), I_{\text {rob }}(p)=I_{\text {rob }}\left(f_{p}\right)$, where $T_{S}$ represents the sampling period.
The optimal DFT method for Gaussian noise averages $x(n) \exp \left(-j 2 \pi f_{p} n T_{S}\right)=x(n) \exp (-j 2 \pi p n / N)$ for each frequency (i.e., the standard DFT):

$$
\begin{aligned}
\hat{X}_{\mathrm{ST}}(p)= & \hat{X}_{\mathrm{ST}}\left(f_{p}\right)=\frac{1}{N} \sum_{n=1}^{N} x(n) \exp \left(\frac{-j 2 \pi p n}{N}\right) \\
= & \operatorname{mean}\left\{x(n) \exp \left(\frac{-j 2 \pi p n}{N}\right)\right\} \\
= & \operatorname{mean}\left\{\operatorname{Re}\left[x(n) \exp \left(\frac{-j 2 \pi p n}{N}\right)\right]\right\} \\
& +j \operatorname{mean}\left\{\operatorname{Im}\left[x(n) \exp \left(\frac{-j 2 \pi p n}{N}\right)\right]\right\},
\end{aligned}
$$

where $\mathrm{Re}$ and Im denote real and imaginary parts of a complex-valued number. Generally, the RDFT can be described as

$$
\begin{aligned}
R_{\text {rob }}(p) & =T\left\{\operatorname{Re}\left[x(n) \exp \left(\frac{-j 2 \pi p n}{N}\right)\right]\right\}, \\
I_{\text {rob }}(p) & =T\left\{\operatorname{Im}\left[x(n) \exp \left(\frac{-j 2 \pi p n}{N}\right)\right]\right\},
\end{aligned}
$$

where $T\{\cdot\}$ denotes applied robust estimator. It should be mentioned that there are many different robust estimators. Hence, such an estimator, $T\{\cdot\}$, should be carefully chosen since the resulting properties of the corresponding RDFT methods depend on $T\{\cdot\}$.

The RDFT-based signal processing methods should provide accurate results in the following cases.

(1) A signal, $s(n)$, can be either "smooth" or "highfrequency" (note that in the latter case the standard low-pass nonlinear filters cause signal degradation [9]).

(2) Noise can resemble the Gaussian case or it might have heavy-tailed PDF [28]. Furthermore, limited a priori information about noise statistics may exist, but not necessarily.

By applying the robust operators in (4), we obtain significant improvements in comparison to the mean operators for heavy-tailed noise environments $[13,14]$. The presence of only one or a few samples corrupted by impulse(s) can lead 


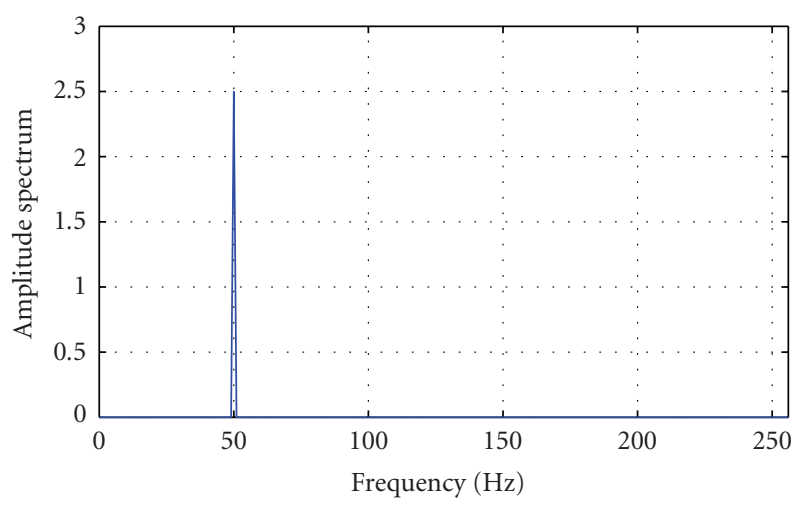

(a)

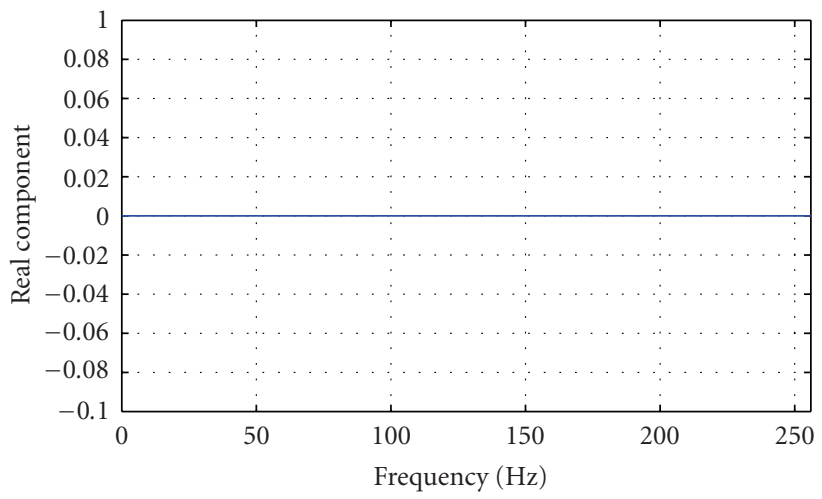

(c)

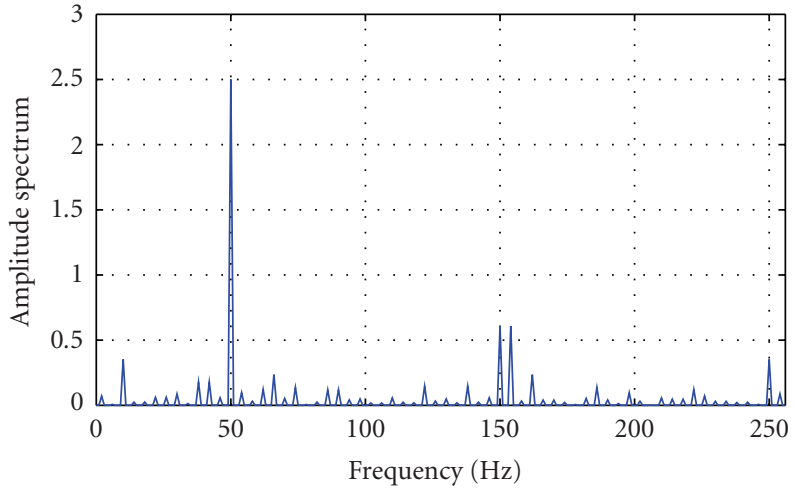

(b)

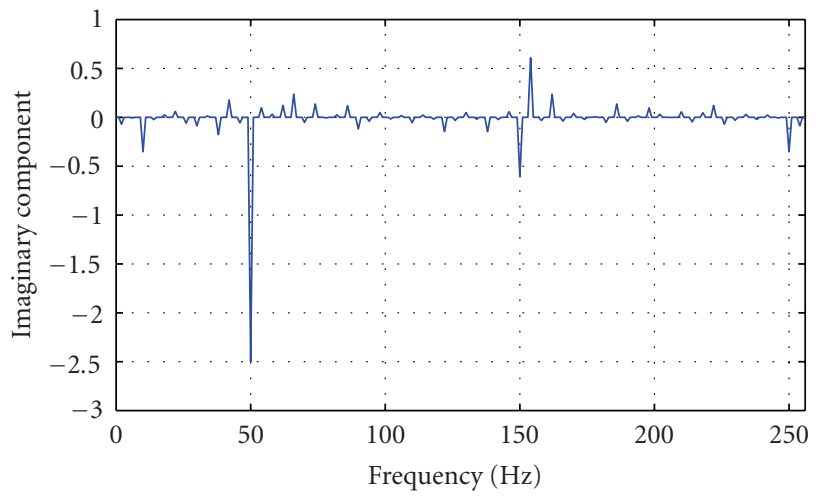

(d)

FIGURE 2: Amplitude spectrum estimates of noise-free signal $s_{1}(n)$ obtained by standard DFT (a) and RDFT method based on MED (b); $R_{\mathrm{MED}}(p)(\mathrm{c})$ and $I_{\mathrm{MED}}(p)(\mathrm{d})$.

to considerable deterioration of $\hat{X}_{\mathrm{ST}}(p)$. The RDFT methods suppress these outliers and achieve more accurate estimates of the nonnoisy signal spectrum. Therefore, the major task is to obtain $\hat{X}_{\mathrm{rob}}(p)=R_{\mathrm{rob}}(p)+j I_{\mathrm{rob}}(p)$ as close to

$$
\begin{aligned}
S(p)= & \operatorname{mean}\left\{\operatorname{Re}\left[s(n) \exp \left(\frac{-j 2 \pi p n}{N}\right)\right]\right\} \\
& +j \text { mean }\left\{\operatorname{Im}\left[s(n) \exp \left(\frac{-j 2 \pi p n}{N}\right)\right]\right\} \\
= & R_{S}(p)+j I_{S}(p)
\end{aligned}
$$

as possible for each $f_{p}$.

However, it is not an easy task. To illustrate this problem, consider a simple case of a real-valued harmonic signal $s_{1}(n)=s_{1}\left(n T_{S}\right)=A \sin \left(2 \pi F n T_{S}\right)$ where $F=$ $50 \mathrm{~Hz}, A=5$, corrupted by zero-mean Gaussian noise $v(n)$. Figure 2 represents the amplitude spectrum $|S(p)|$ obtained by the standard DFT and its estimates $\left|\hat{S}_{\text {MED }}(p)\right|$, $R_{\mathrm{MED}}(p)$, and $I_{\mathrm{MED}}(p)$ evaluated by the RDFT method, where $T\{\ldots\}$ is the sample median (MED). It can be clearly seen that the estimate $\left|\hat{S}_{\mathrm{MED}}(p)\right|$ differs from the conventional spectrum $|S(p)|$ due to distortions introduced in $I_{\mathrm{MED}}(p)$ (for a test signal $A \cos \left(2 \pi F n T_{S}\right.$ ) distortions appear in $R_{\mathrm{MED}}(p)$ ). To further understand the appearance of these undesirable disturbances, we consider the histograms of the samples $R(p, n)=\{x(n) \cos (2 \pi p n / N)\}$ and $I(p, n)=$ $\{-x(n) \sin (2 \pi p n / N)\}$ for two frequencies: $f_{p}=F$ where there are no distortions introduced by RDFT method and $f_{p}=3 F=150 \mathrm{~Hz}$ where $|S(p)|$ is considerably distorted (see Figure 2(b)). Values of the corresponding estimators $R_{S}(p), I_{S}(p), R_{\mathrm{MED}}(p)$, and $I_{\mathrm{MED}}(p)$ are marked at histograms by solid and dashed lines, respectively. For each histogram we have determined the percentile coefficient of kurtosis (PCK) [29] (for mathematical definition see Table 5). This parameter characterizes the distribution tail heaviness. For Gaussian PDF it is equal to 0.26 while for distributions with heavier tails, PCK is smaller. For $S \alpha S$ processes, the PCK value decreases when $\alpha$ value becomes smaller.

In parallel, the median of absolute deviations (MADs) from the sample median has been calculated to characterize the data scale (see Table 5). MAD is used instead of the standard deviation since considered distributions might have heavy tails. This will be demonstrated later.

Let us analyze the histograms for $f_{p}=F$ shown in Figures 3(a) and 3(b). Both represented histograms are symmetric with respect to their location parameter (LP). Then, $R_{\mathrm{MED}}(p)=R_{S}(p)$ and $I_{\mathrm{MED}}(p)=I_{S}(p)$. Consider the second case with $f_{p}=3 F$. The corresponding histograms represented in Figures 3(c) and 3(d) show that the $R(p, n)$ distribution is again symmetric with respect to its LP whereas the histogram of $I(p, n)$ is asymmetric. Then $I_{S}(p)$ and 


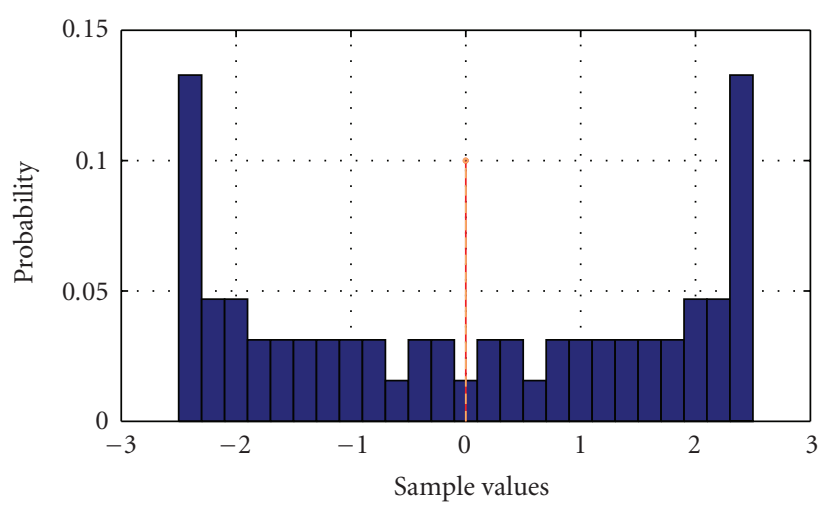

(a)

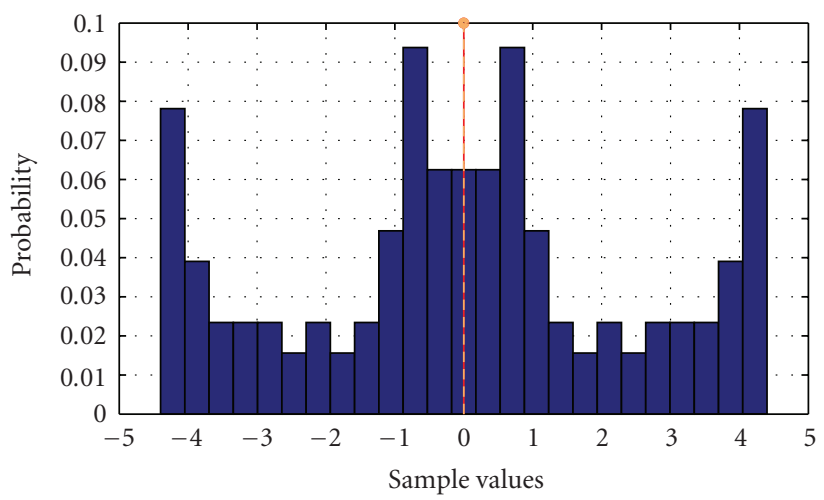

(c)

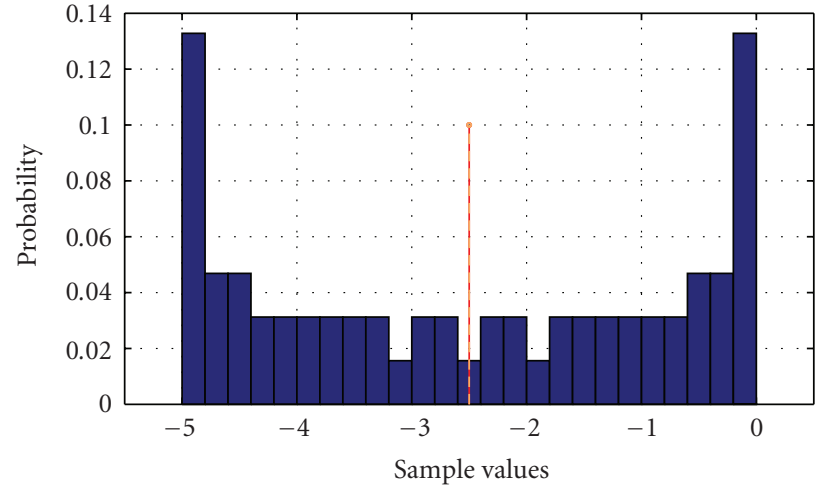

(b)

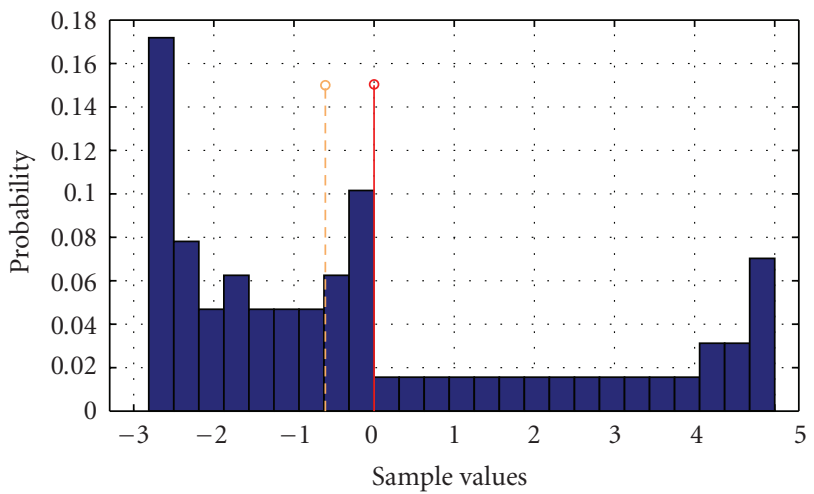

(d)

FIGURE 3: Histograms of $R(p, n)$ (left column) and $I(p, n)$ (right column) for $f_{p}=F\left((\mathrm{a})\right.$ and $(\mathrm{b})$ ) $\mathrm{PCK}_{\mathrm{RE}}=\mathrm{PCK}_{\mathrm{IM}}=0.37, \mathrm{MAD}_{\mathrm{RE}}=$ $\mathrm{MAD}_{\mathrm{IM}}=1.77((\mathrm{a})$ and $(\mathrm{b}))$; for $f_{p}=3 F((\mathrm{c})$ and $(\mathrm{d})), \mathrm{PCK}_{\mathrm{RE}}=0.19, \mathrm{MAD}_{\mathrm{RE}}=1.47(\mathrm{c}), \mathrm{PCK}_{\mathrm{IM}}=0.28$, and $\mathrm{MAD}_{\mathrm{IM}}=1.71(\mathrm{~d})$.

$I_{\mathrm{MED}}(p)$ differ as in the cases of other asymmetric PDFs like Rayleigh distribution [30]. Contrary to the previous case, the values of PCK and MAD are also different for PDFs of RE and IM components.

The fact that $I_{\mathrm{MED}}(p) \neq I_{S}(p)$ for some frequencies introduces distortions in a signal spectrum estimate. Then, the spectrum estimate distortions lead to the distortions of the filtered signal in temporal domain. Recall that the filtered signal is obtained by using the inverse standard DFT of the robust estimate $\hat{S}_{\text {rob }}(p)$.

Aforementioned problem is common for some other robust estimators. For example, if one uses $\alpha$-trimmed mean estimator (ATM), distortions in the output (filtered) signal are observed as well. They are clearly demonstrated in Figure 4. This spectral distortion effect is undesirable, and the main goal is its minimization in the RDFT-based signal processing methods.

Now consider the case of a signal corrupted by Gaussian noise for two different values of the standard deviation, $\sigma_{G}=$ 1 and $\sigma_{G}=10$. The histograms for two frequencies $f_{p}=F$ and $f_{p}=3 F$ for $\sigma_{G}=1$ are presented in Figures 5(a)5(d). As it can be seen, the distributions in Figures 5(a) and 5 (b) are light tailed but not symmetric; $R_{\mathrm{MED}}(p) \neq R_{S}(p)$, $I_{\mathrm{MED}}(p) \neq I_{S}(p)$. The PDFs depicted in Figures 5(c) and 5(d) are also asymmetric but with heavier tails. Distribution scales are in both cases approximately the same.
The histograms for $f_{p}=F$ and $f_{p}=3 F$ for $\sigma_{G}=10$ are presented in Figures 5(e)-5(h), respectively. MAD values have considerably increased in comparison to previous cases $\left(\sigma_{G}=1\right)$. All distributions are almost symmetric, and they have heavy tails. Thus, the presence of input noise has considerably changed PDF properties of the considered data samples in comparison to the earlier considered case of noise absence. Since the distributions are heavy tailed, the use of robust estimators can give us some benefits.

Now, we analyze the accuracy of the RDFT methods and compare with the standard DFT. The MSE of the spectrum estimate is calculated as

$$
\begin{gathered}
\operatorname{MSE}_{i}=\frac{1}{M} \sum_{m=1}^{M} \operatorname{MSE}_{i}(m), \\
\operatorname{MSE}_{i}(m)=\frac{1}{N} \sum_{p=0}^{N-1}\left|\hat{X}_{i}^{m}(p)-S(p)\right|^{2},
\end{gathered}
$$

where $\hat{X}_{i}^{m}(p)$ is the estimate of the spectrum obtained by the $i$ th method for the $m$ th signal realization; $i$ denotes the investigated method where the standard DFT and the RDFT method based on MED are considered; $M=100$ is the number of realizations.

The results are presented in Table 1. As expected, the MSE for the standard DFT (MSE ${ }_{\text {ST }}$ ) increases proportionally 
TABLE 1: MSE for signal $s_{1}(n)$ spectrum estimates obtained by standard DFT and RDFT method based on MED for the case of Gaussian noise with different values of $\sigma_{G}$.

\begin{tabular}{lccccccc}
\hline \multicolumn{7}{c}{$\sigma_{G}$} \\
\hline & 0 & 0.5 & 1 & 2 & 4 & 8 & 0 \\
\hline MSE $_{\text {ST }}$ & 0 & 0.0005 & 0.0020 & 0.0078 & 0.0313 & 0.1240 & 0.1949 \\
MSE $_{\text {MED }}$ & 0.0055 & 0.0052 & 0.0052 & 0.0061 & 0.0123 & 0.0350 & 0.0547 \\
\hline
\end{tabular}

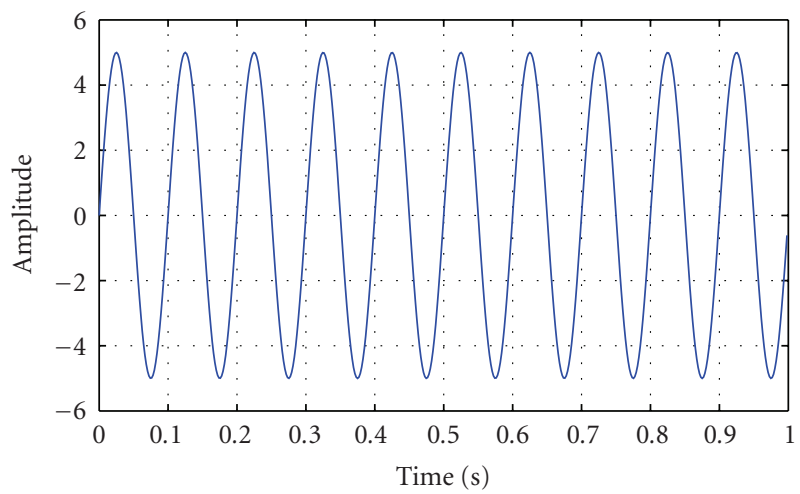

(a)

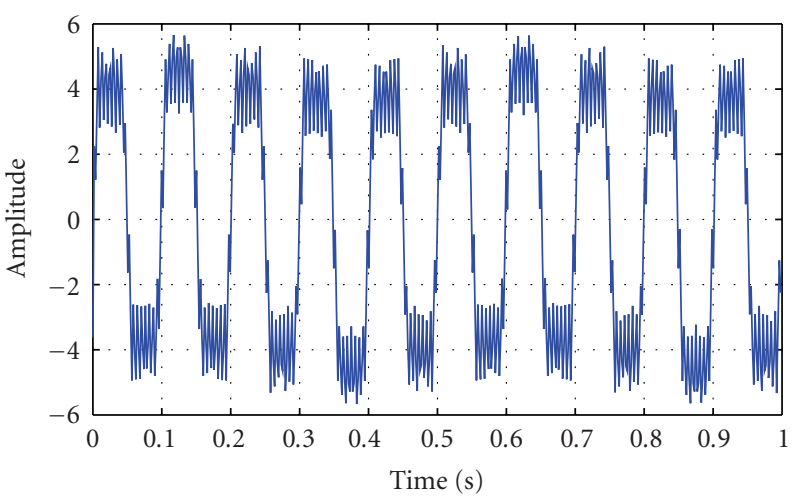

(b)

FIGURE 4: Example of distortions introduced by ATM method of RDFT: the noise-free harmonic signal $(F=10 \mathrm{~Hz})(\mathrm{a})$ and its estimate obtained using inverse DFT of $\hat{S}_{\mathrm{ATM}}(p)(\mathrm{b})$.

to $\sigma_{G}^{2}$. For RDFT method based on MED, MSEMED is not equal to zero even for $\sigma_{G}=0$. This is due to the previously described distortions. However, for large $\sigma_{G}$ (see, for instance, $\sigma_{G}=4$ ), the value of $\mathrm{MSE}_{\mathrm{MED}}$, is considerably smaller than $\mathrm{MSE}_{\mathrm{ST}}$, that is, the median RDFT form gives better estimation of the DFT. Thus, advantages of the RDFT based processing become apparent for rather important practical case of low input SNR even for the Gaussian input noise.

Next, consider another marginal case where the input sample is equal to noise without signal. This scenario is particularly important when dealing with signal detection where it is desirable to decrease noise level at the output.

Figure 6 represents a realization of heavy-tailed noise (modeled as a product of two independent zero-mean white Gaussian variables with $\sigma_{G}=1$ ) and its estimate obtained as the inverse DFT of $\hat{X}_{\mathrm{MED}}(p)$. Clearly, impulses have been removed, and noise has become less intensive. Here, we would like to emphasize that the distribution of modulated noise $\nu(n) \sin \left(2 \pi f_{p} T_{S} n / N\right)$ is not equal to the distribution of $v(n)[31]$.

Consider now a more complicated case of an FM signal. As a case study, let us analyze signal $s_{2}(n)=$ $A \sin \left(2 \pi F(n) n T_{S}\right)$ where $A=5, F(n)=a n T_{S}$, for $n=0, F=$ 0 , for $n T_{S}=1 \mathrm{sec}$., $F=50 \mathrm{~Hz}(a=25)$. Figures $7(\mathrm{a})-7(\mathrm{c})$ represent $|S(p)|, R_{S}(p)$ and $I_{S}(p)$ for the considered noisefree signal. There is a range of frequencies (over $100 \mathrm{~Hz}$ ) for which $|S(p)|, R_{S}(p)$, and $I_{S}(p)$ are practically equal to zero. The estimates $\left|\hat{S}_{\mathrm{MED}}(p)\right|, R_{\mathrm{MED}}(p)$, and $I_{\mathrm{MED}}(p)$, evaluated by RDFT method based on the MED, are demonstrated in Figures 7(d)-7(f). Again, the RDFT method introduces distortions.

Consider the histograms of $R(p, n)$ and $I(p, n)$ for two frequencies. Let us first analyze the histograms for the frequency $f_{p}=32 \mathrm{~Hz}$ which is inside the signal spectrum band. Just for this frequency, the spectrum distortions are quite large. As demonstrated in Figures 8(a)-8(b), we obtain asymmetric distributions, inducing a lack of overlap between the mean and MED values. Note that these distributions for the FM signal $s_{2}(n)$ considerably differ from those ones for the harmonic signal $s_{1}(n)$ shown in Figure 3.

Next, let us analyze the case when Gaussian noise is present. Histograms for $f_{p}=32 \mathrm{~Hz}$ are shown in Figures 8(c) and $8(\mathrm{~d})\left(\sigma_{G}=10\right)$. The distributions have become heavy tailed with wider limits in comparison to the distributions shown in Figures 8(a) and 8(b). The obtained MSEs of spectrum estimates for noise-free and noisy signal $s_{2}(n)$ are presented in Table 2. For the standard DFT, the MSE increases proportionally to $\sigma_{G}$ as in the previous case. For the MED method of RDFT, MSE ${ }_{\text {MED }}$ is not equal to zero even for $\sigma_{G}=0$ due to introduced distortions. For large $\sigma_{G}$ (e.g., $\left.\sigma_{G}=10\right)$, the $\mathrm{MSE}_{\mathrm{MED}}$ value is almost four times smaller than the $\mathrm{MSE}_{\mathrm{ST}}$ value, that is, the RDFT approach achieves a more accurate estimate of the spectrum for low values of the input SNR.

Concluding the analysis, we can state the following. First, there are practical cases when even the simplest RDFT method yields more accurate results than the standard DFT. However, there are also cases when RDFT introduces considerable distortions. Taking into account the above-mentioned 


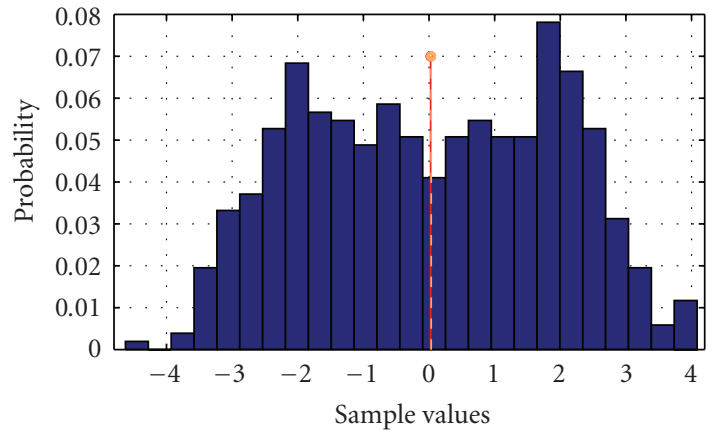

(a)

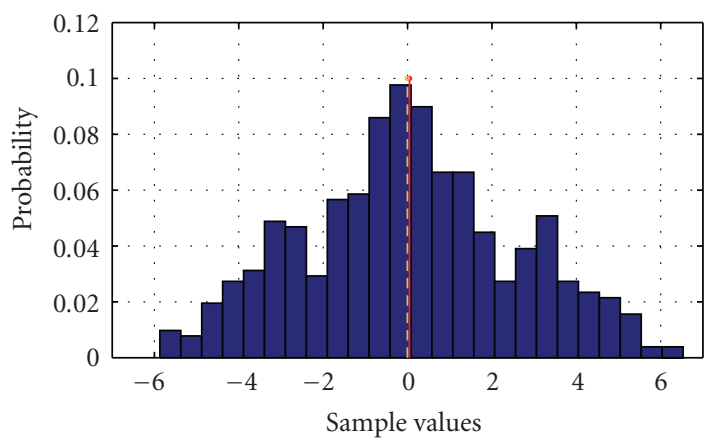

(c)

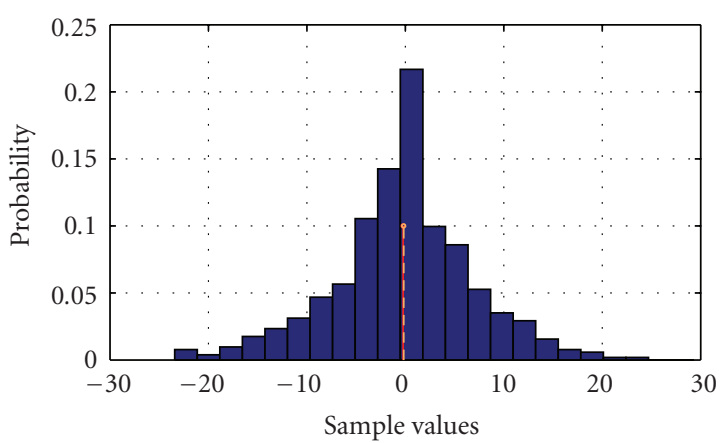

(e)

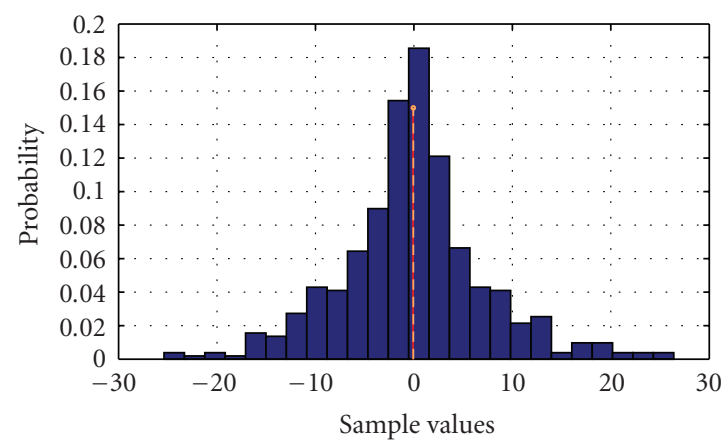

(g)

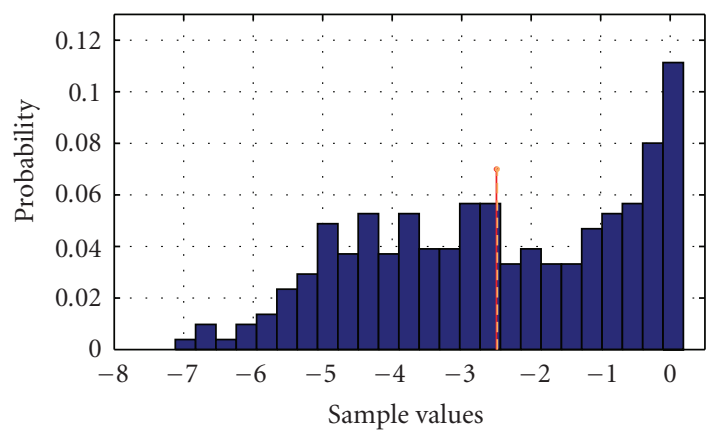

(b)

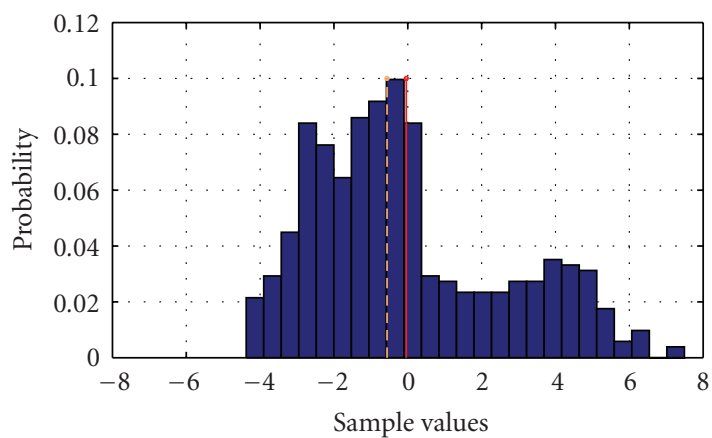

(d)

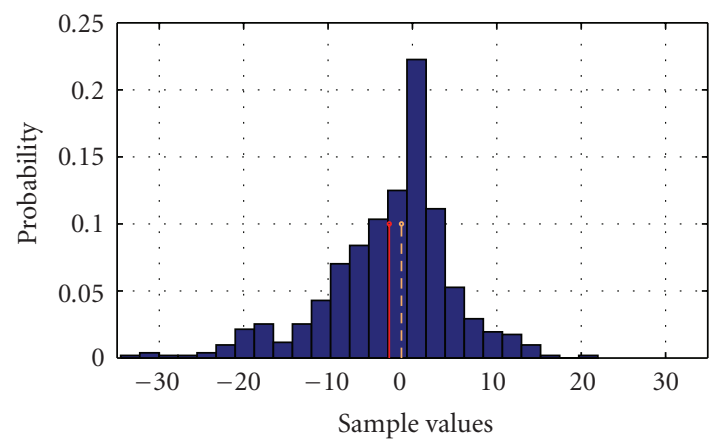

(f)

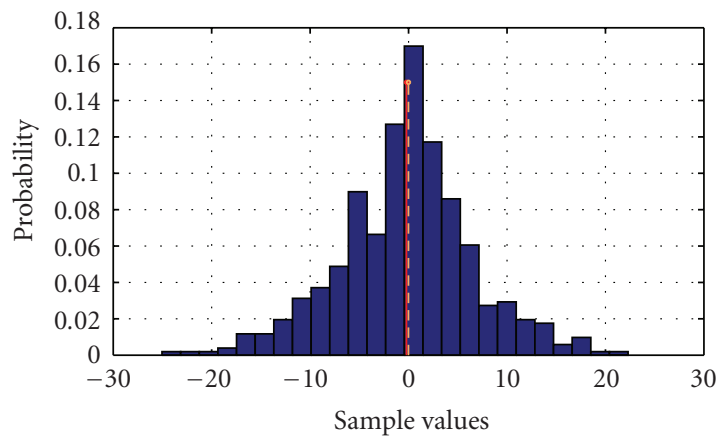

(h)

FIGURE 5: Histograms of $R(p, n)$ (left column) and $I(p, n)$ (right column) for: $f_{p}=F, \sigma_{G}=1 ; \mathrm{PCK}_{\mathrm{RE}}=0.330, \mathrm{MAD}_{\mathrm{RE}}=1.65, \mathrm{PCK} \mathrm{IM}=0.341$, $\operatorname{MAD}_{\mathrm{IM}}=1.73((\mathrm{a})$ and $(\mathrm{b})) ; f_{p}=3 F, \sigma_{G}=1 ; \mathrm{PCK}_{\mathrm{RE}}=0.243, \mathrm{MAD}_{\mathrm{RE}}=1.67, \mathrm{PCK}_{\mathrm{IM}}=0.252, \mathrm{MAD}_{\mathrm{IM}}=1.68((\mathrm{c})$ and $(\mathrm{d})) ; f_{p}=F$, $\sigma_{G}=10 ; \mathrm{PCK}_{\mathrm{RE}}=0.209, \mathrm{MAD}_{\mathrm{RE}}=3.77, \mathrm{PCK}_{\mathrm{IM}}=0.231, \mathrm{MAD}_{\mathrm{IM}}=3.94((\mathrm{e})$ and (f) $) ; f_{p}=3 F, \sigma_{G}=10 ; \mathrm{PCK}_{\mathrm{RE}}=0.186, \mathrm{MAD}_{\mathrm{RE}}=3.46$, $\mathrm{PCK}_{\mathrm{IM}}=0.237, \mathrm{MAD}_{\mathrm{IM}}=3.92((\mathrm{~g})$ and $(\mathrm{h}))$. 
TABLE 2: MSE for signal $s_{2}(n)$ spectrum estimates obtained by standard DFT and RDFT method based on MED for the case of Gaussian noise with different values of $\sigma_{G}$.

\begin{tabular}{lccccccr}
\hline & \multicolumn{7}{c}{$\sigma_{G}$} \\
\hline & 0 & 0.5 & 1 & 2 & 4 & 8 & 10 \\
\hline MSE $_{\text {ST }}$ & 0 & 0.00039 & 0.00163 & 0.00671 & 0.0270 & 0.1077 & 0.1725 \\
MSE $_{\text {MED }}$ & 0.00543 & 0.00555 & 0.00610 & 0.00845 & 0.0154 & 0.0317 & 0.0459 \\
\hline
\end{tabular}

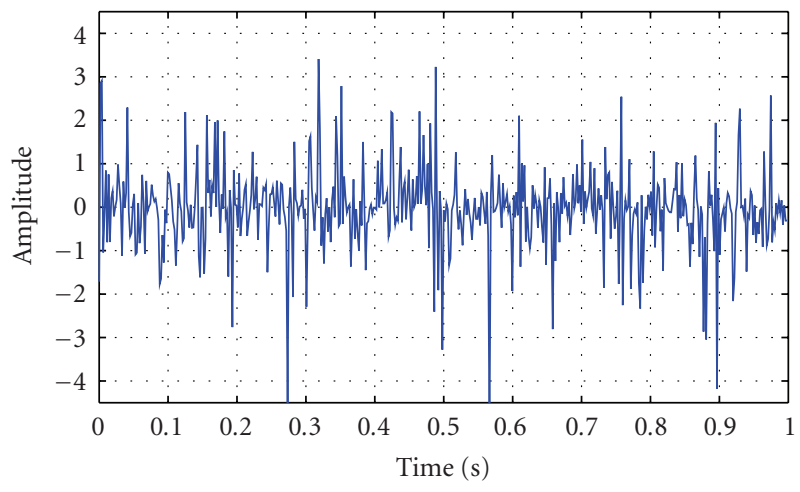

(a)

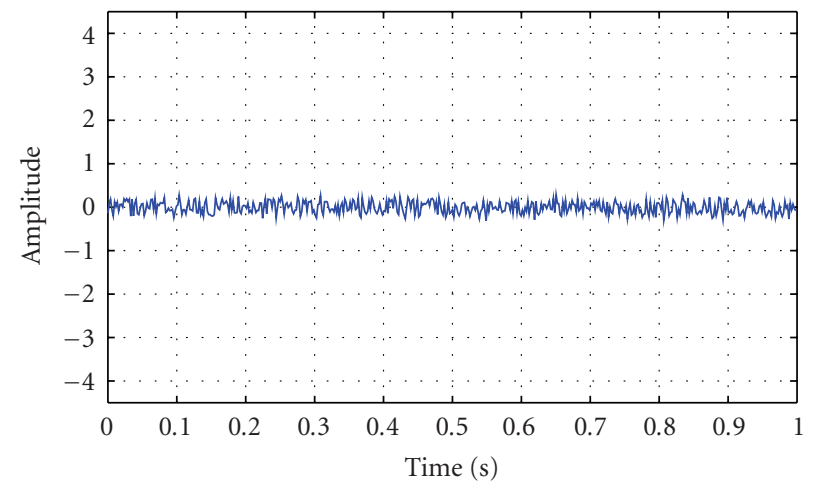

(b)

FIGURE 6: The realization of the original heavy-tailed noise $\left(\sigma_{G}=1\right)$ and its estimate obtained after inverse DFT of $\hat{X}_{\mathrm{MED}}(p)$.

properties, the spectrum estimate obtained by RDFT methods can be represented as

$$
\begin{aligned}
\hat{X}_{\mathrm{rob}}(p)= & R_{\mathrm{rob}}(p)+j I_{\mathrm{rob}}(p) \\
= & {\left[R_{S}(p)+\Delta R(p)\right] } \\
& +j\left[I_{S}(p)+\Delta I(p)\right]
\end{aligned}
$$

where the requirement for estimators is to provide

$$
|\Delta R(p)| \longrightarrow \min ,|\Delta I(p)| \longrightarrow \min
$$

for each $p$. Note that if (9) is satisfied, noise is suppressed in the temporal domain after the inverse DFT. Therefore, we analyze the estimators meeting these criteria in Section 3.

\section{Adaptive Robust Estimators and their Properties}

There is a profound theory of LP estimation for data samples with symmetric distributions $[5,29]$. It allows obtaining optimal maximum likelihood (ML) and L-estimators for data realizations with a priori known PDFs. However, distributions of modulated signal samples for each considered frequency in RDFT data processing approach are unknown in advance.

There are also methods for robust mode finding based on adaptive myriad or interquantile estimators [31, 32]. However, $R_{S}(p)$ and $I_{S}(p)$ can be considerably different from modes of the corresponding distributions (see examples in Figure 5). There are also other adaptive estimators, for example, [33], that are mainly intended on processing data samples with heavy-tailed PDFs.
In Section 2, we analyzed histograms $R(p, n)$ and $I(p, n)$ for particular frequencies. To gain further understanding of the desirable properties of the RDFT estimators we analyze scale and tail heaviness for these data samples for all spectral frequencies of FM signal $s_{2}(n)$ corrupted by non-Gaussian noise (Figure 9). As a practical example, consider noise modeled as the $S \alpha \mathrm{S}$ process with $\alpha=1.5$ and $\gamma=1$. Note that such a value of $\alpha$ is typical for atmospheric noise [33]. As it is seen in Figure 9(b), the signal is corrupted with several impulses.

The plots of $\operatorname{MAD}_{\mathrm{RE}}(p), \operatorname{MAD}_{\mathrm{IM}}(p), \operatorname{PCK}_{\mathrm{RE}}(p)$ and $\operatorname{PCK}_{\mathrm{IM}}(p)$ for $\alpha=1.5$ and $\gamma=1$ are given in Figure 10. Each value of PCK and MAD obtained for each pth frequency is averaged over 10 realizations to get more consistent values. Practically for all frequencies (except the 256th), $\operatorname{MAD}_{\mathrm{RE}}(p)$ and $\operatorname{MAD}_{\mathrm{IM}}(p)$ are approximately equal. The values of $\operatorname{PCK}_{\mathrm{RE}}(p)$ and $\operatorname{PCK}_{\mathrm{IM}}(p)$ (except $p=N / 2=256$ ) are approximately equal as well. They do not significantly differ from value 0.267 typical for a Gaussian PDF. This indicates that the distribution for all these frequencies is approximately equal to Gaussian.

A special case is the distributions for the $p=N / 4$ which equals $f_{p}=128 \mathrm{~Hz}$ for the considered example. Both MAD and PCK are close to zero in this case. The reason is that for this frequency there are many values of $x(n) \cos (2 \pi p n / N)$ and $x(n) \sin (2 \pi p n / N)$ that are equal to zero. If $p=N / 4$, then $2 \pi N n / 4 N=\pi n / 2$, and for each odd $n$, the values of $\cos (2 \pi p n / N)$ are equal to 0 . The same holds for all even $n$ for $\sin (2 \pi p n / N)$.

Now consider the case when $\alpha=1.5$ and $\gamma=4$, that is, stronger intensity noise. The plots of $\operatorname{MAD}_{\mathrm{RE}}(p)$, $\operatorname{MAD}_{\mathrm{IM}}(p), \mathrm{PCK}_{\mathrm{RE}}(p)$, and $\mathrm{PCK}_{\mathrm{IM}}(p)$ are given in Figure 11. For all frequencies (except the 256th), $\operatorname{MAD}_{\mathrm{RE}}(p)$ and $\operatorname{MAD}_{\operatorname{IM}}(p)$ are approximately equal but they have slightly 


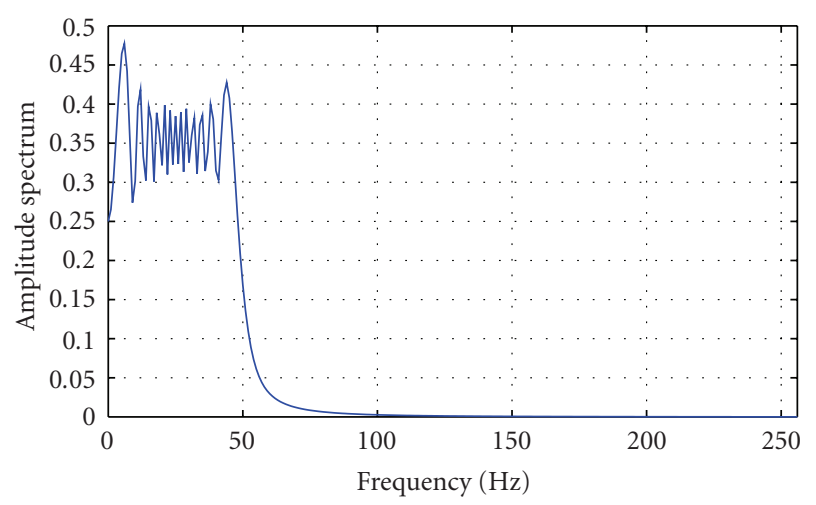

(a)

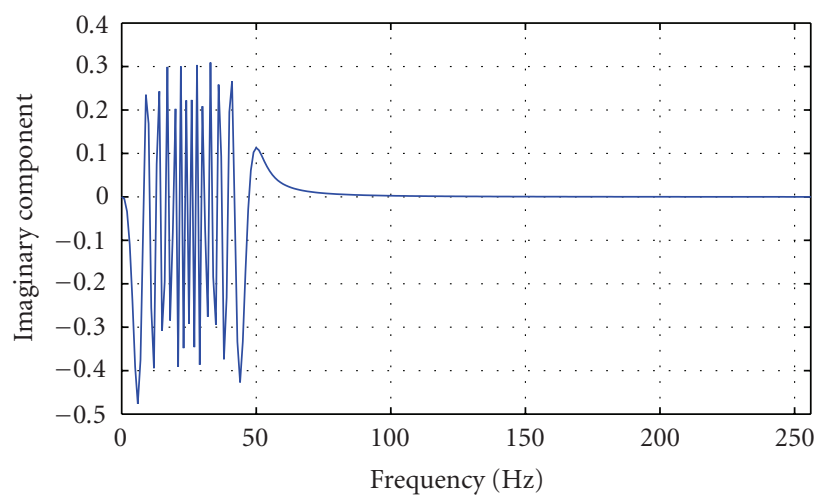

(c)

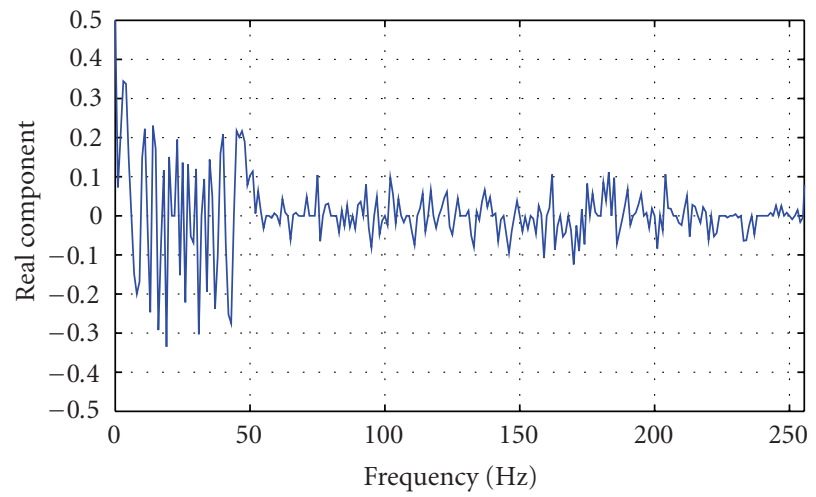

(e)

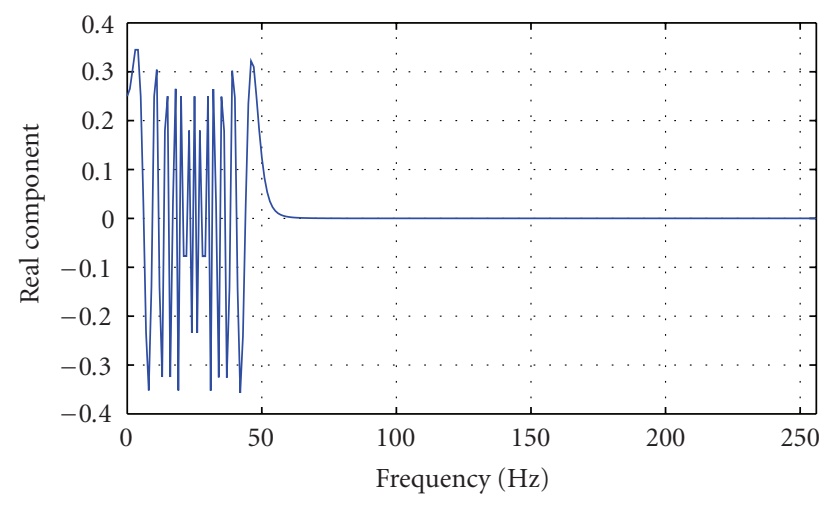

(b)

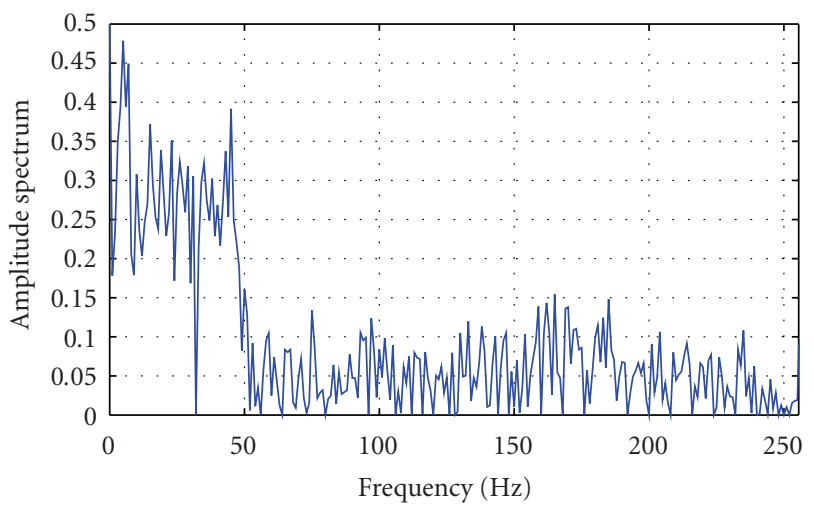

(d)

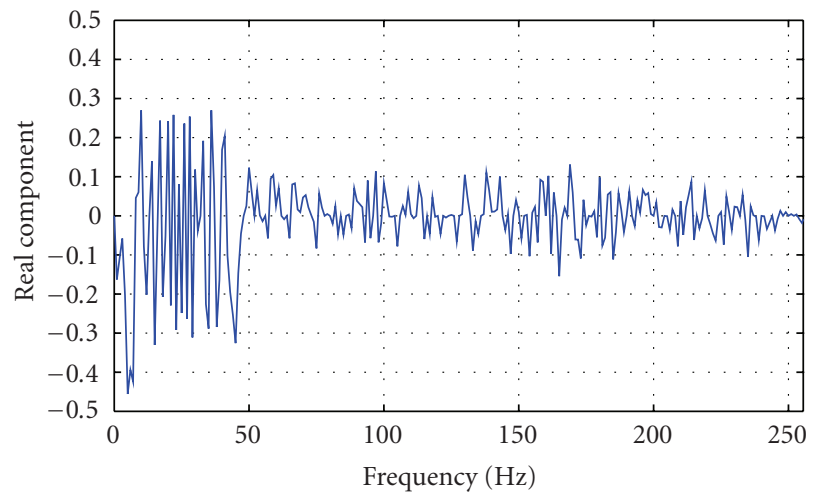

(f)

FIGURE 7: Amplitude spectrum of noise-free FM signal $s_{2}$ ( $n$ ) (left column), and its RE (central column) and IM (right column) components obtained by standard DFT ((a)-(c)) and MED-form of RDFT ((d)-(f)).

increased in comparison to those presented in Figure 10. The values $\operatorname{PCK}_{\mathrm{RE}}(p)$ and $\mathrm{PCK}_{\mathrm{IM}}(p)$ (except $p=N / 2=256$ ) are approximately equal, but have decreased compared to Figure 10 . They are now approximately equal to 0.2 , that is, the PDF sufficiently differs from the Gaussian distribution and has heavy tails. This implies that even when noise tail heaviness is known in advance, the characteristics of noise in $R(p, n)$ and $I(p, n)$ described by $\operatorname{PCK}_{\mathrm{RE}}(p)$ and $\operatorname{PCK}_{\mathrm{IM}}(p)$ can vary depending upon SNR. Then, one has to adapt to distribution characteristics of $R(p, n)$ and $I(p, n)$ in order to provide accurate spectral estimation and denoising.
As it follows from the analysis, the estimator should be robust to outliers but also close to the sample mean for PDFs without heavy tails (see Figures 3 and 5).

Two examples of such estimators are Wilcoxon (WE) and Hodges-Lehmann (HL) estimators [34, 35] (for details see Table 5). Their statistical properties are quite similar, but the latter one requires less computations especially for a large sample size $N$. Therefore, we consider the HL estimator below. Note that these robust estimators produce the LP estimates close to the sample mean [36] for the Gaussian PDF. 


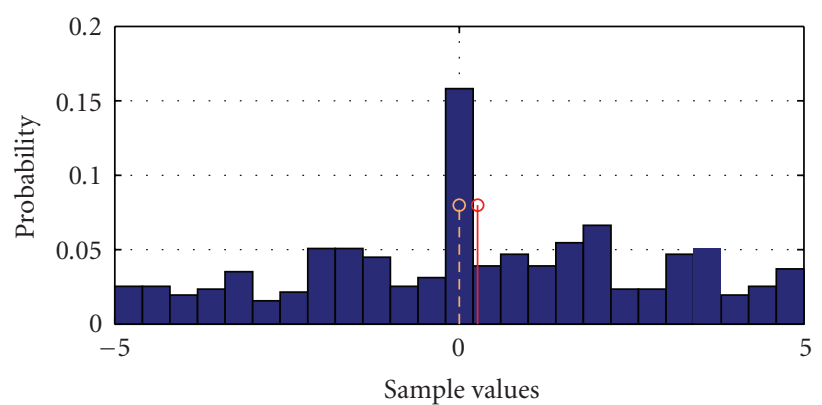

(a)

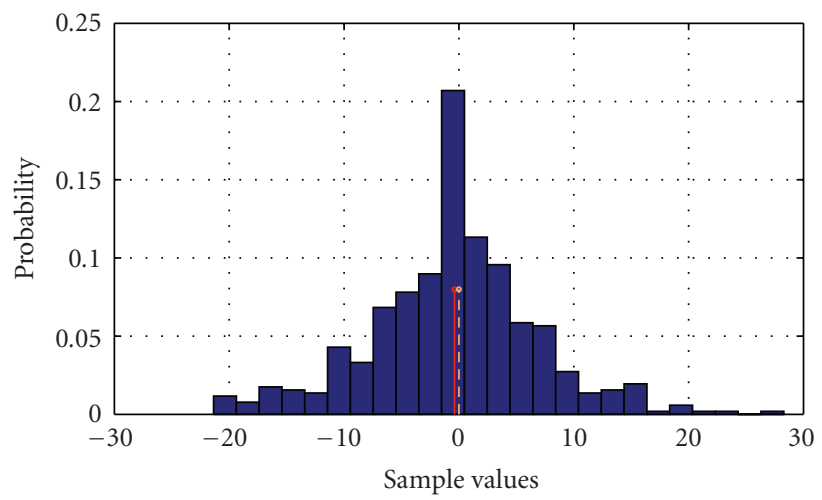

(c)

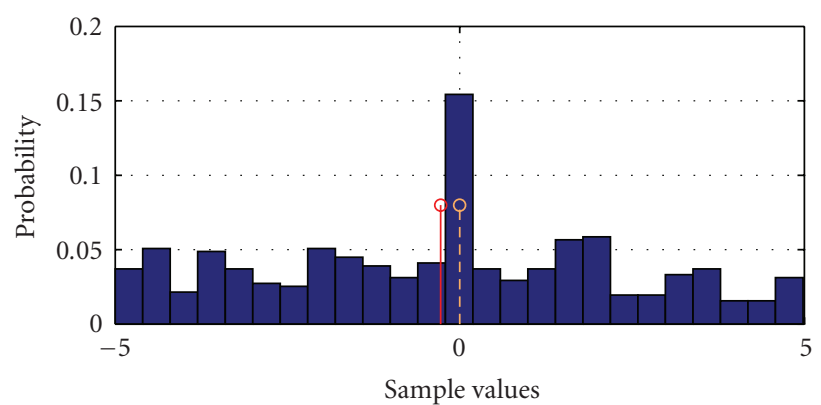

(b)

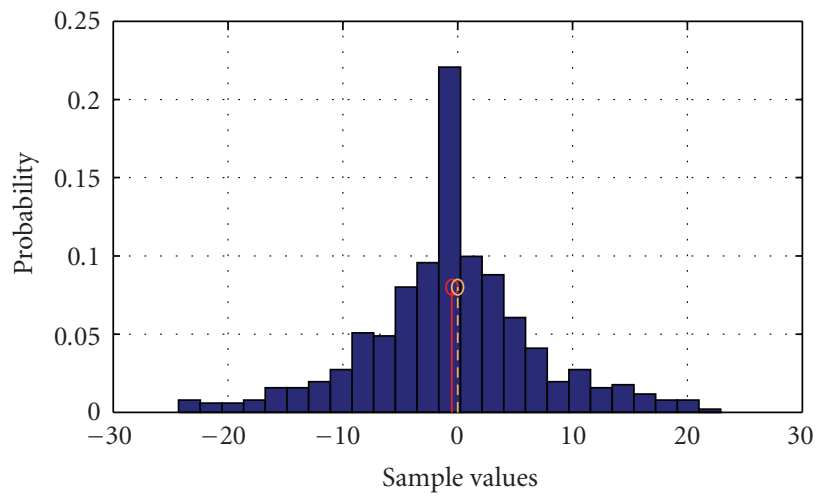

(d)

Figure 8: Histograms of $R(p, n)$ (left column) and $I(p, n)$ (right column) for noise-free ((a) and (b)) and noisy $((\mathrm{c})$ and $(\mathrm{d}))$ FM signal $s_{2}(n)$ for $f_{p}=32 \mathrm{~Hz}, \mathrm{PCK}_{\mathrm{RE}}=0.250, \mathrm{MAD}_{\mathrm{RE}}=1.838, \mathrm{PCK}_{\mathrm{IM}}=0.261, \mathrm{MAD}_{\mathrm{IM}}=1.84$ (noise-free case), $\mathrm{PCK}_{\mathrm{RE}}=0.219, \mathrm{MAD}_{\mathrm{RE}}=3.75$, $\mathrm{PCK}_{\mathrm{IM}}=0.211$, and $\mathrm{MAD}_{\mathrm{IM}}=3.74$ (noisy case).

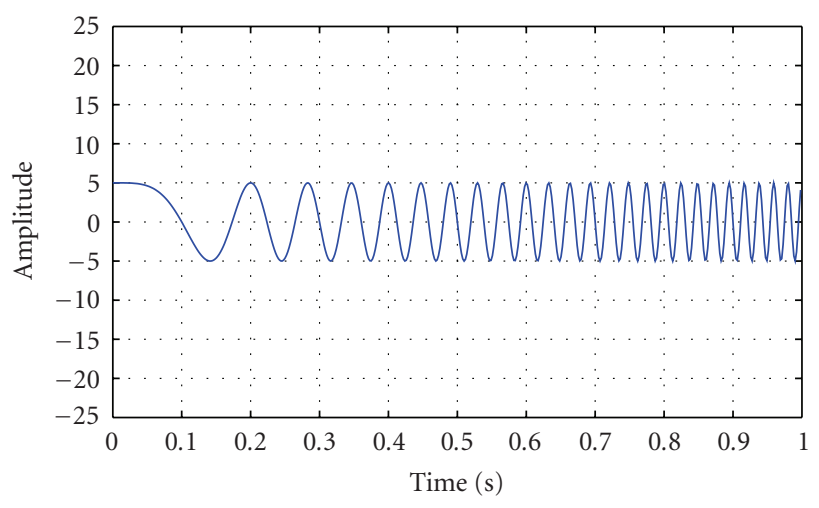

(a)

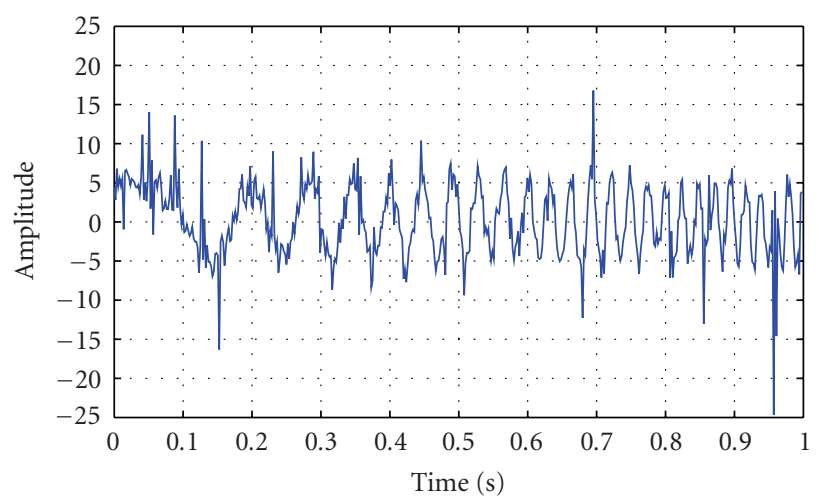

(b)

FIGURE 9: The test noise-free FM signal $s_{2}(n)$ (a) and its realization corrupted by $\mathrm{S} \alpha \mathrm{S}$ noise with $\alpha=1.5$ and $\gamma=1$ (b).

Another estimator obeying the desired property is the ATM $[5,7,29]$ with the trimming parameter $\beta=N_{\beta} / N$ where $N_{\beta}$ defines the number of outer trimmed elements for a data sample of size $N$ (see Table 5). A well-known property of ATM is to be equal to the MED if $\beta \rightarrow 0.5$ and to the sample mean if $\beta \rightarrow 0$ [7].

A third estimator capable of producing an estimate close to the mean for nonnoisy signals is the sample myriad (MYR) under the condition that its tunable parameter $K$ is considerably larger than the standard deviation of the data sample [37]. In practice, it is sufficient to set $K$ greater than the data sample standard deviation multiplied by 3 $[38,39]$. The definition of the MYR is represented in Table 5. Main properties of the MYR estimator can significantly vary depending on the value of $K$. In particular, for a relatively small $K$, MYR is able to perform as an efficient mode finder for both symmetric and asymmetric distributions $[6,30]$.

Therefore, we conclude that there are several robust estimators which under certain conditions are capable of producing a desired behavior for data samples. However, 


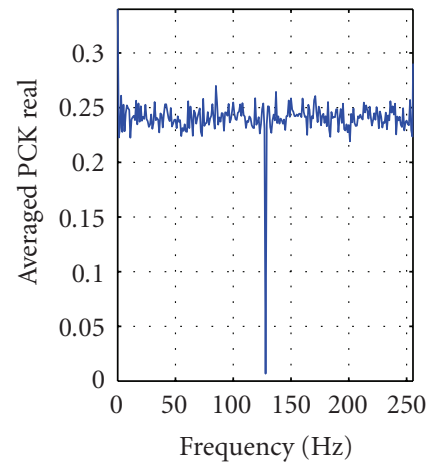

(a)

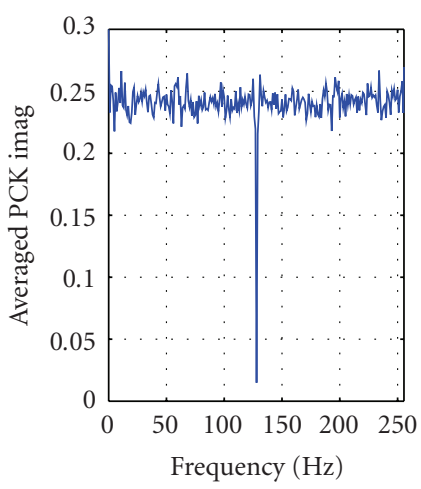

(c)

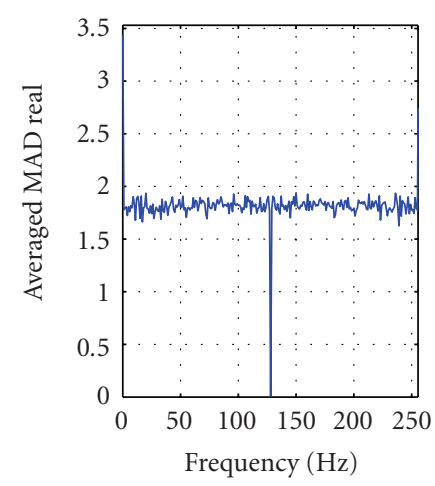

(b)

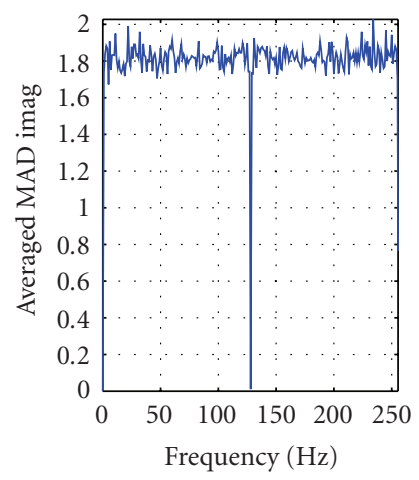

(d)

Figure 10: The plots of $\operatorname{PCK}_{\mathrm{RE}}(p)(\mathrm{a})$ and $\mathrm{PCK}_{\mathrm{IM}}(p)(\mathrm{c}), \mathrm{MAD}_{\mathrm{RE}}(p)(\mathrm{b})$ and $\mathrm{MAD}_{\mathrm{IM}}(p)(\mathrm{d})$ for FM signal $s_{2}(n)$ corrupted by $\mathrm{S} \alpha \mathrm{S}$ noise with $\alpha=1.5$ and $\gamma=1$.

for modulated signal samples we have different statistics for $R(p, n)$ and $I(p, n)$ for each frequency (see Figures 5 and 8 ). Then, a question is how an estimator can adapt to such situations of a priori unknown or changing properties of underlying distributions? Obviously, we need some sort of adaptive RDFT estimators.

One of these solutions is Taguchi's adaptive alphatrimmed mean estimator (AATME) [40]. A second solution [17] is the adaptive estimator that presumes simple hard switching (ADHS) between the MED (applied if an estimated PCK for a data sample is smaller than a preset threshold $\psi$ ) and HL estimator (used otherwise). The recommended value of $\psi$ is about 0.2 (for details see Table 5).

The PCK is used also in the censored mean adaptive estimator (CENS) [20]. However, it also exploits a robust estimate of data scale, namely, MAD for a considered data sample. The estimator CENS exploits the facts that the PCK for Gaussian distribution is approximately equal to 0.26 and the standard deviation equals $\sigma_{G}=1.483 \mathrm{MAD}$. Then, the formed neighborhood $D$ in case of Gaussian noise approximately corresponds to $3 \sigma_{G}$ neigborhood of the mean (for details see Table 5).

In order to understand how distortions are reduced or removed in the CENS-based RDFT method, let us consider the following example. According to the plots in Figure 10, the values of MAD are approximately equal to $2, \mathrm{PCK}$ is about 0.25 , and then one obtains $D \approx 9$ (see expression for $D$ in Table 5). Taking into account that $\operatorname{med}\{R(p, n)\}$ and $\operatorname{med}\{I(p, n)\}$ are about 0 (see Figure 8), the neighborhood limits are from about -9 till about +9 . The values belonging to this neighborhood are averaged. Thus, the estimator performs similarly to the sample mean with respect to the values that approximately fit to reasonably narrow limits (see Figure 8$)$. It can be shown that all values $R(p, n)$ and $I(p, n)$ participate in averaging for the histograms presented in Figures 3, 5(a)-5(d), and 8(a) and 8(b). At the same time, there are outer trimmed values for the histograms in Figures 5 and 8, that is, for situations when noise is non-Gaussian and/or intensive. This is desired for a robust estimator to be used in the RDFT framework.

There are also two adaptive myriad estimators (AM1 and AM2) that have been developed recently [38, 39]. The first one estimates $\gamma$ and $\alpha$ supposing that an underlying distribution is $\mathrm{S} \alpha \mathrm{S}$. Estimations of $\gamma$ and $\alpha$ are based on calculations of MAD and PCK. Then the optimal $K$ is calculated for given estimates $\hat{\alpha}$ and $\hat{\gamma}$ using established dependences $[38,39]$. The second adaptive myriad estimator AM2 directly calculates the optimal $K$ using evaluated values of MAD and PCK for the data sample at hand (for details concerning AM1 and AM2 see Table 5).

Next, we determine the performance of these estimators. To partly address this problem, consider $\mathrm{S} \alpha \mathrm{S}$ distributions. 


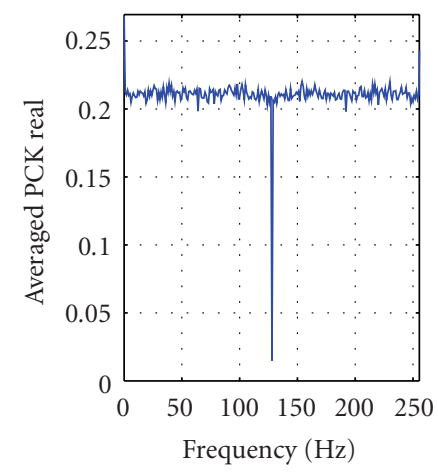

(a)

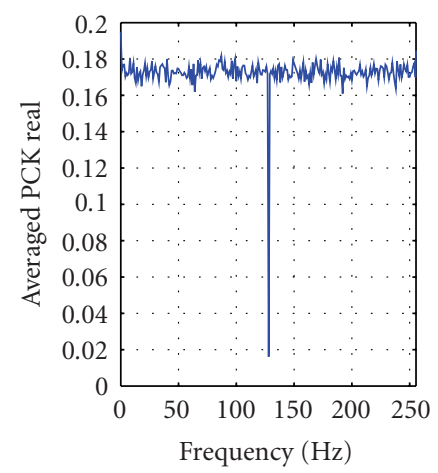

(c)
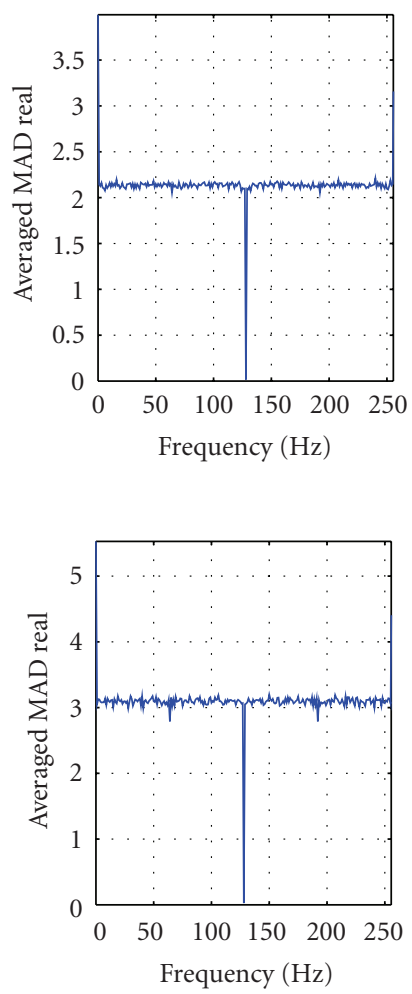
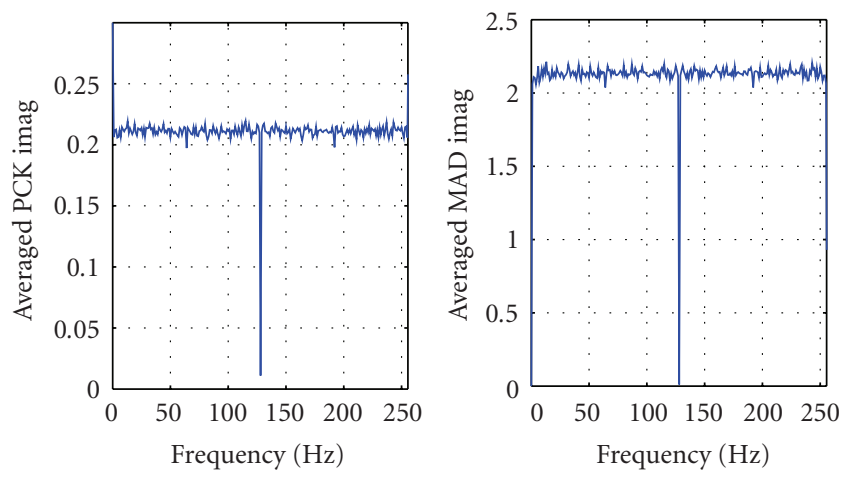

(b)
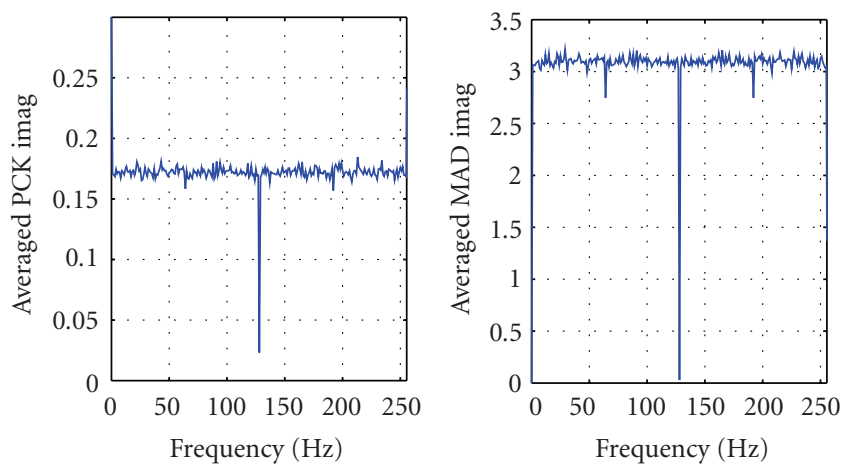

(d)

Figure 11: The plots of $\operatorname{PCK}_{\mathrm{RE}}(p), \operatorname{MAD}_{\mathrm{RE}}(p)$ (left column) and $\mathrm{PCK}_{\mathrm{IM}}(p), \mathrm{MAD}_{\mathrm{IM}}(p)$ (right column) for FM signal $s_{2}(n)$ corrupted by $\operatorname{SiS}$ noise with $\alpha=1.5$ (upper string) and $\alpha=1$ (bottom string) $(\gamma=4)$.

There is an approach that allows determining an asymptotic variance of the optimal M-estimator of LP for each distribution from $S \alpha S$ family [41]. Variance of the myriad estimator with optimally set $K$ can be analytically determined as well.

Moreover, it is possible to determine optimal weights for the L-estimator. They are presented in Figure 12(a) for data sample of size $N=64$. For $\alpha=2$ that corresponds to Gaussian PDF, the sample mean is the optimal L-estimator (all order statistics have the same weights equal to $1 / N$ ). For small $\alpha$, order statistics with $n \approx N / 2$ have considerably larger weights than others, that is, MED is the quasi optimal L-estimator. These results serve as good explanations of operation principles for the AATME and CENS estimators.

Now, we analyze the variances of the considered estimators for the LP estimation. They are presented as dependences on $\alpha$ for $\gamma=1$ and $N=512$ in Figure 12(b). As it can be seen, all adaptive estimators perform well. In the worst cases, variances for them are only 30\% larger than for the optimal M-estimator. Adaptive myriad estimators provide the best performance for small $\alpha$. If the data sample size $N$ decreases, relative performance of all adaptive estimators becomes poorer. The reason is due to the fact that obtained values of the MAD and PCK are less accurate for smaller $N$.

The main conclusion that follows from this analysis is that the adaptive robust estimators seem to be able to provide improvement if they are used in RDFT framework instead of non-adaptive estimators such as the MED or ATM.

\section{Use of the Proposed Estimators in RDFT-Based Signal Processing}

Let us consider the efficiency of the proposed adaptive robust estimators within the RDFT framework of signal processing. Consider MSE of spectral estimates for test signals (TS) $s_{1}(n)$ and $s_{2}(n)$ determined by (6) and (7).

The following RDFT methods have been analyzed:

(1) method based on MED estimator $\left(\widehat{X}_{\mathrm{MED}}\right)$;

(2) method based on ATM estimator with fixed $\beta=0.25$ $\left(\hat{X}_{\mathrm{ATM}}\right)$;

(3) method based on WE estimator $\left(\hat{X}_{\mathrm{WE}}\right)$;

(4) method based on HL estimator $\left(\hat{X}_{\mathrm{HL}}\right)$;

(5) method based on AATME estimator ( $\left.\hat{X}_{\text {AATME }}\right)$;

(6) method based on ADHS estimator $\left(\hat{X}_{\mathrm{ADHS}}\right)$;

(7) method based on CENS estimator ( $\left.\hat{X}_{\mathrm{CENS}}\right)$;

(8) method based on adaptive myriad estimator $\left(\hat{X}_{\mathrm{AM}}\right)$.

The estimators 1-4 are non-adaptive, and the estimators 5- 8 are adaptive. The estimators 5 and 7 are, in fact, special kinds of adaptive alpha-trimmed mean estimators.

The results of the numerical analysis of the considered methods for harmonic TS $s_{1}(n)$ corrupted by zero-mean white additive Gaussian noise are summarized in Table 3. 


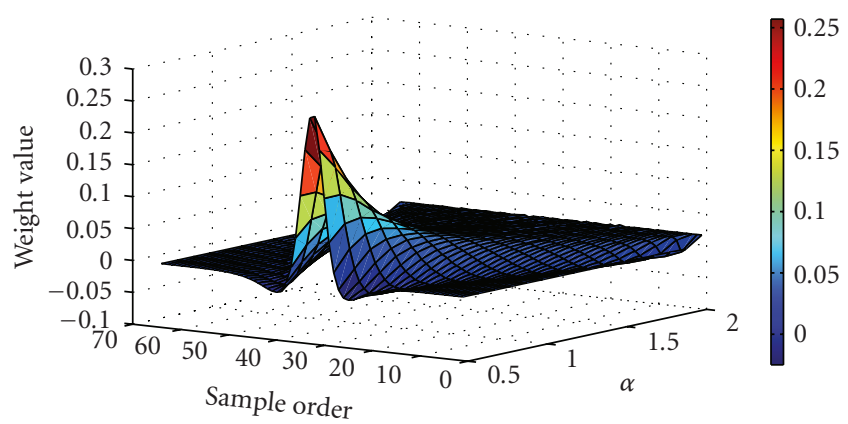

(a)

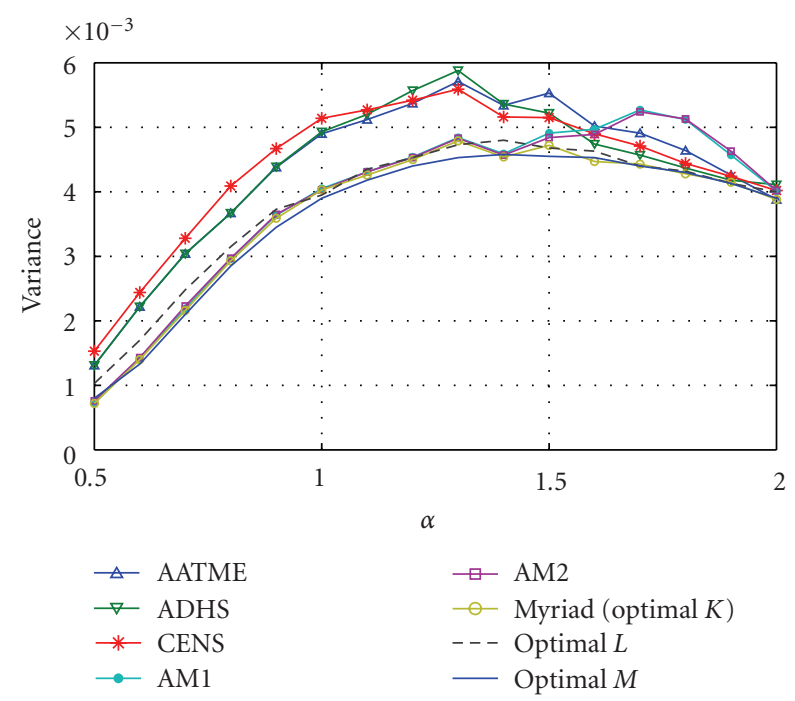

(b)

Figure 12: Optimal weights for L-estimator $(N=64)$ (a) and variance values of LP estimation for the considered adaptive and optimal estimators $(N=512)$ (b) for the $\mathrm{S} \alpha \mathrm{S}$ processes $(\gamma=1)$.

Let us start with the analysis of the noise-free case. All non-adaptive estimators introduce certain distortions. However, for the WE and HL estimators these distortions are considerably smaller than for the standard MED. Adaptive estimators except the CENS also introduce distortions. The performance of other adaptive estimators deteriorates; however, they still achieve more accurate results than the MED.

If the input SNR is large enough (consider $\sigma_{G}=$ 1), the results for the standard DFT and RDFT methods based on the WE and the proposed adaptive estimators are comparable. For other estimators, the negative effect of introduced distortions is larger than the positive effect of noise suppression.

If the input SNR is small (see data for $\sigma_{G}=10$ ), MSE values for all robust forms of DFT (except Taguchi's estimator) are smaller than for the standard DFT. The best results are provided by the MED method; among adaptive estimators the ADHS produces the smallest MSE. Thus, there is no estimator that allows obtaining a minimal MSE for all considered situations. The main advantage of the adaptive robust estimators is that they produce MSE close to minimally reachable for wide ranges of input SNR.

Another set of experiments has been carried out for the FM signal $s_{2}(n)$ corrupted by heavy-tailed noise. Noise has been simulated as a process with $\mathrm{S} \alpha \mathrm{S}$ PDF with $\alpha$ from 0.5 till 2 and fixed $\gamma=1$. The simulation data are collected in Table 4.

Let us start analysis from Gaussian noise case $(\alpha=2)$. An MED form of DFT produces considerable distortions that result in MSE almost three times larger than for standard DFT. Other RDFT methods provide a smaller MSE, especially the methods based on W, HL, and ADHS estimators.

For $\alpha=1.5$, the influence of heavy-tailed noise on the standard DFT becomes significant. All RDFT forms estimate the signal spectrum more accurately than the standard DFT. Again, the RDFT methods based on the WE, HL and ADHS estimators provide the best results. Other adaptive estimators have a slightly worse accuracy. The standard DFT fails for noise with Cauchy PDF $(\alpha=1)$. All adaptive methods are almost as accurate as the non-adaptive methods.

Finally, if $\alpha=0.5$, the standard DFT fails, and the MED method of RDFT achieves the best performance among nonadaptive forms. The best adaptive methods are based on Taguchi's adaptive estimator or CENS in this case. Other adaptive methods perform reasonably well.

Thus, when statistical properties of noise are unknown, the main advantage of adaptive estimators used in RDFT framework is that they produce accuracy close to reachable best values.

To demonstrate this advantage, consider two examples. Figure 13(a) shows the amplitude spectrum estimate obtained by the standard DFT. As depicted, noise masks the spectrum of the signal and this estimate resembles an estimate of the white noise spectrum. The spectrum estimate obtained by the MED method of RDFT is demonstrated in Figure 13(b). Here the signal spectrum in the frequency band $F \leq 50 \mathrm{~Hz}$ is clearly seen but it is distorted (the thin line shows the RDFT spectrum estimate, and the thick solid line relates to the true spectrum of FM signal $\left.s_{2}(n)\right)$. Amplitudes of spectrum estimates for $F>50 \mathrm{~Hz}$ have sufficiently decreased due to applying the robust estimator. Finally, the adaptive CENS estimator estimates more accurately the signal component spectrum for $F \leq 50 \mathrm{~Hz}$ (see the plot in Figure 13(c)). This simple example demonstrates two advantages of the RDFT methods. First, these methods achieve higher noise suppression in the frequency range where noise-free signal spectrum is practically equal to zero. Second, they also achieve more accurate estimates in the frequency range where the noise-free signal spectrum essentially differs from zero.

Then, if a signal spectrum is estimated based on the RDFT, it becomes possible to carry out denoising with using ideal low-pass filter in spectral domain that has $K(p)=1$ if $f_{p} \leq f_{\text {upper }}$ and 0 otherwise. Let us set the upper frequency $f_{\text {upper }}$ equals $100 \mathrm{~Hz}$ for the considered TS $s_{2}(n)$. The input signal corrupted by noise with Cauchy PDF and $\gamma=1$ is presented in Figure 14(a). The output signal is obtained by the application of RDFT method based on CENS estimator, 
TABLE 3: MSE values of spectrum estimation for TS $s_{1}(n)$ corrupted by additive Gaussian noise.

\begin{tabular}{|c|c|c|c|c|c|c|c|}
\hline$\sigma_{G}$ & 0 & 0.5 & 1 & 2 & 4 & 8 & 10 \\
\hline$\hat{X}_{\mathrm{ST}}$ & 0 & 0.00049 & 0.00192 & 0.00782 & 0.0309 & 0.249 & 0.194 \\
\hline$\hat{X}_{\mathrm{MED}}$ & 0.00545 & 0.00537 & 0.00545 & 0.00613 & 0.0115 & 0.0370 & 0.058 \\
\hline$\hat{X}_{\text {ATM }}$ & 0.00362 & 0.00390 & 0.00478 & 0.00896 & 0.0250 & 0.0862 & 0.133 \\
\hline$\hat{X}_{\mathrm{WE}}$ & 0.00058 & 0.00090 & 0.00197 & 0.00626 & 0.0218 & 0.0844 & 0.132 \\
\hline$\hat{X}_{\mathrm{HL}}$ & 0.00199 & 0.00211 & 0.00284 & 0.00690 & 0.0237 & 0.0913 & 0.142 \\
\hline$\hat{X}_{\text {AATME }}$ & 0.00498 & 0.00559 & 0.00610 & 0.00864 & 0.0309 & 0.1250 & 0.194 \\
\hline$\hat{X}_{\mathrm{ADHS}}$ & 0.00199 & 0.00212 & 0.00285 & 0.00685 & 0.0174 & 0.0592 & 0.097 \\
\hline$\hat{X}_{\text {CENS }}$ & 0 & 0.00051 & 0.00199 & 0.00913 & 0.0301 & 0.1033 & 0.156 \\
\hline$\widehat{X}_{\mathrm{AM}}$ & $8.81 \cdot 10^{-7}$ & 0.00048 & 0.00207 & 0.00809 & 0.0239 & 0.0829 & 0.127 \\
\hline
\end{tabular}

TABLE 4: MSE values of spectrum estimation for FM signal $s_{2}(n)$ corrupted by noise $(\gamma=1)$.

(a)

\begin{tabular}{lcccccccc}
\hline$\alpha$ & 0.5 & 0.6 & 0.7 & 0.8 & 0.9 & 1 & 1.1 \\
$\hat{X}_{\mathrm{ST}}$ & $\infty$ & $\infty$ & $\infty$ & $\infty$ & $\infty$ & $\infty$ & $\infty$ & $\mathbf{0}$ \\
$\hat{X}_{\mathrm{MED}}$ & $\mathbf{0 . 0 1 7 7}$ & $\mathbf{0 . 0 1 5 2}$ & $\mathbf{0 . 0 1 3 3}$ & $\mathbf{0 . 0 1 1 7}$ & $\mathbf{0 . 0 1 1 2}$ & $\mathbf{0 . 0 1 0 2}$ & $\mathbf{0 . 0 0 9 5}$ & 0.0090 \\
$\hat{X}_{\mathrm{ATM}}$ & 0.0352 & 0.0270 & 0.0207 & 0.0168 & 0.0143 & 0.0117 & 0.0102 & 0.0088 \\
$\hat{X}_{\mathrm{WE}}$ & 0.0452 & 0.0325 & 0.0236 & 0.0181 & 0.0147 & 0.0114 & $\mathbf{0 . 0 0 9 5}$ & $\mathbf{0 . 0 0 7 7}$ \\
$\hat{X}_{\mathrm{HL}}$ & 0.0504 & 0.0366 & 0.0264 & 0.0204 & 0.0165 & 0.0127 & 0.0105 & 0.0084 \\
$\hat{X}_{\mathrm{AATME}}$ & $\mathbf{0 . 0 1 3 3}$ & $\mathbf{0 . 0 1 1 6}$ & $\mathbf{0 . 0 1 1 1}$ & $\mathbf{0 . 0 1 0 1}$ & $\mathbf{0 . 0 0 9 4}$ & 0.0089 & 0.0085 \\
$\hat{X}_{\text {ADHS }}$ & 0.0230 & 0.0197 & 0.0162 & 0.0127 & 0.0104 & 0.0084 & $\mathbf{0 . 0 0 7 1}$ & 0.0230 \\
$\hat{X}_{\mathrm{CENS}}$ & 0.0179 & 0.0151 & 0.0131 & 0.0111 & 0.0096 & $\mathbf{0 . 0 0 8 2}$ & 0.0073 \\
$\hat{X}_{\mathrm{AM}}$ & 0.0211 & 0.0182 & 0.0161 & 0.0149 & 0.0121 & 0.0092 & 0.0179 \\
\hline
\end{tabular}

(b)

\begin{tabular}{lcccccccc}
\hline$\alpha$ & 1.3 & 1.4 & 1.5 & 1.6 & 1.7 & 1.8 & 1.9 \\
$\hat{X}_{\text {ST }}$ & 0.2929 & 0.0978 & 0.36609 & 0.03078 & 0.00927 & 0.00822 & 0.00797 & 0.00249 \\
$\hat{X}_{\text {MED }}$ & 0.0087 & 0.0080 & 0.00773 & 0.00752 & 0.00726 & 0.00714 & 0.00557 & 0.00653 \\
$\hat{X}_{\text {ATM }}$ & 0.0077 & 0.0067 & 0.00597 & 0.00550 & 0.00498 & 0.00447 & 0.00660 & 0.00367 \\
$\hat{X}_{\text {WE }}$ & $\mathbf{0 . 0 0 6 6}$ & $\mathbf{0 . 0 0 5 3}$ & $\mathbf{0 . 0 0 4 4 3}$ & $\mathbf{0 . 0 0 3 9 6}$ & $\mathbf{0 . 0 0 3 3 8}$ & $\mathbf{0 . 0 0 2 8 5}$ & $\mathbf{0 . 0 0 3 9 3}$ & $\mathbf{0 . 0 0 2 0 9}$ \\
$\hat{X}_{\text {HL }}$ & 0.0071 & 0.0057 & 0.00472 & 0.00418 & 0.00359 & 0.00300 & 0.00462 & 0.00215 \\
\hline$\hat{X}_{\text {AATME }}$ & $\mathbf{0 . 0 1 1 6}$ & 0.0077 & 0.00714 & 0.00667 & 0.00616 & 0.00561 & 0.00726 & 0.00481 \\
$\hat{X}_{\text {ADHS }}$ & 0.0197 & $\mathbf{0 . 0 0 5 7}$ & $\mathbf{0 . 0 0 4 7 4}$ & $\mathbf{0 . 0 0 4 2 0}$ & $\mathbf{0 . 0 0 3 6 0}$ & $\mathbf{0 . 0 0 3 0 0}$ & $\mathbf{0 . 0 0 4 6 2}$ & $\mathbf{0 . 0 0 2 1 6}$ \\
$\hat{X}_{\text {CENS }}$ & 0.0151 & 0.0062 & 0.00535 & 0.00480 & 0.00419 & 0.00361 & 0.00497 & 0.00274 \\
$\hat{X}_{\text {AM }}$ & 0.0182 & 0.0072 & 0.00585 & 0.00502 & 0.00425 & 0.00373 & 0.00493 & 0.00277 \\
\hline
\end{tabular}

ideal low-pass filter, and standard inverse DFT. It is shown in Figure 14(b). Obviously, excellent denoising is provided. The impulse noise is removed, but also we achieved the suppression of non-impulsive noise components. This can be confirmed by MAE values

$$
\operatorname{MAE}=\frac{1}{N} \sum_{n=1}^{N}|\hat{x}(n)-x(n)|,
$$

where $\hat{x}(n)$ denotes the reconstructed signal obtained by RDFT filtering approach. MAE = 5.505 for the noisy signal shown in Figure 14(a) while for the denoised signal MAE = 2.147 without low-pass filtering, and $\mathrm{MAE}=1.417$ with lowpass filtering.

\section{Conclusions, Other Related Work, and Perspectives}

The general overview of the RDFT-based signal processing approach is presented, and the requirements for robust estimators are formulated. Several non-adaptive and adaptive estimators are considered. It has been shown that the main advantage of adaptive estimators is the robustness in wide sense. They also provide a possibility to improve efficiency of signal processing (spectrum estimation, denoising) methods to potentially reachable limits for wide range of noise parameters. In particular, the RDFT-based on adaptive estimators can be useful for low input SNRs even if noise is Gaussian. Their application becomes even more practical if noise is non-Gaussian and heavy tailed. Adaptive estimators 


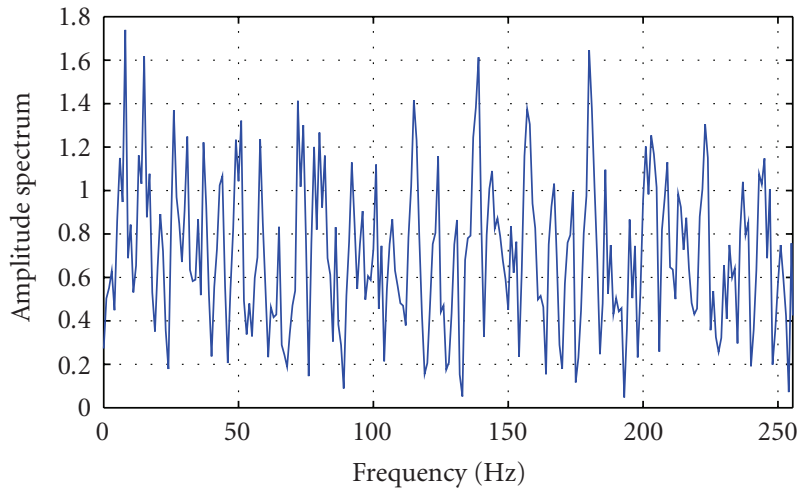

(a)

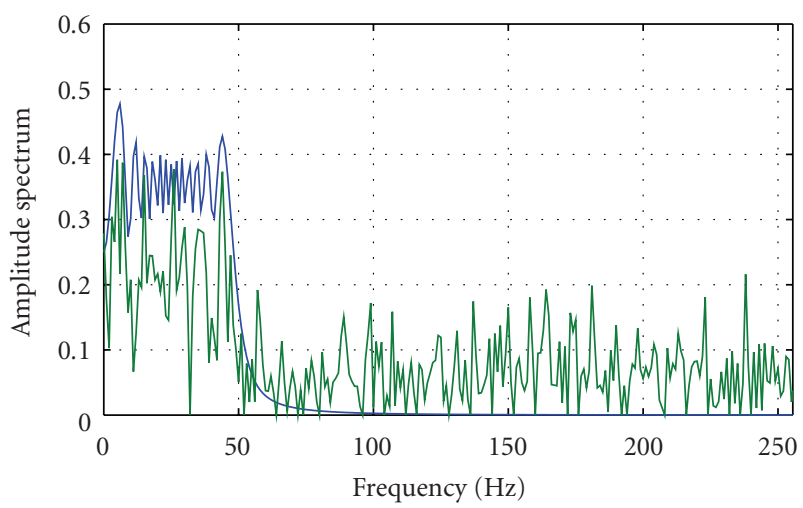

(b)

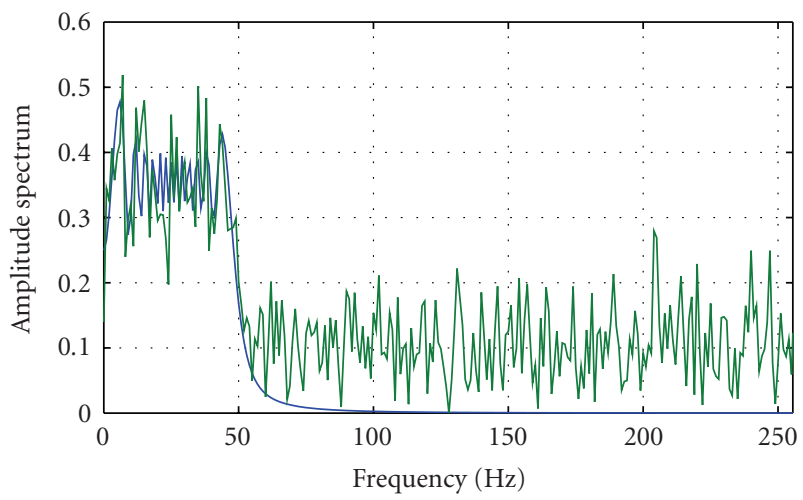

(c)

FIGURE 13: Amplitude spectrum estimates for FM signal $s_{2}(n)$ corrupted by noise with Cauchy PDF $(\gamma=1)$ obtained by the standard DFT (a), MED-method of RDFT (b) and RDFT method based on CENS (c).

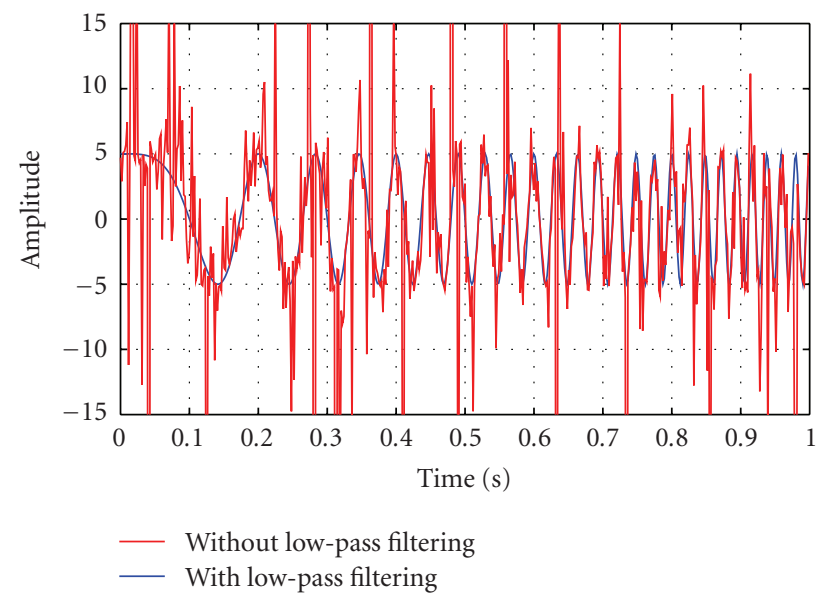

(a)

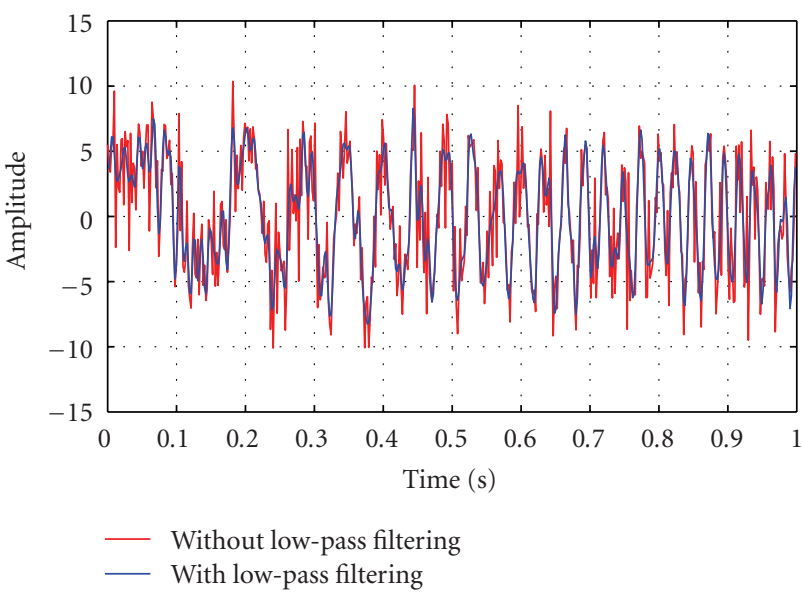

(b)

FIGURE 14: The TS $s_{2}(n)$ corrupted by noise with Cauchy PDF $(\gamma=1)$ (a) and its estimate obtained by RDFT filtering approach based on CENS-method (b).

CENS and ADHS seem to be the best practical choice. All presented analysis was done for harmonic and FM realvalued signals. However, the same steps are also valid for complex-valued input data.
In this overview, we have concentrated on RDFT applications for spectral analysis and filtering. However, it is worth stressing that the RDFT methods can also be applied in other spectral analysis tasks such as the 
TABLE 5

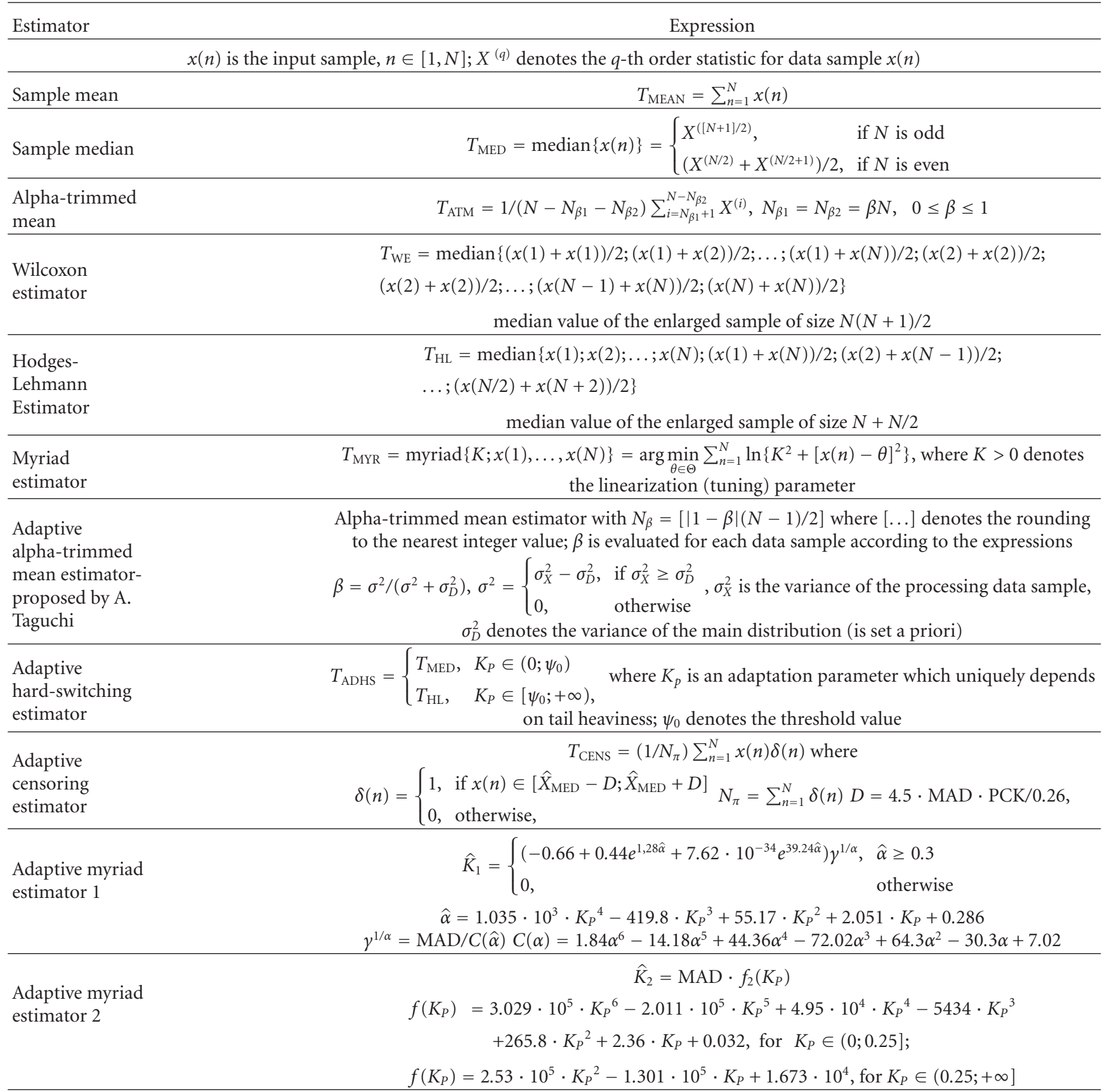

Percentile coefficient of kurtosis

Median absolute

deviation from

median

time-frequency analysis for deriving Wigner-Ville or other distributions [21, 42] and estimation of signal parameters [26].

Also note that robust estimators can be useful for data processing based on other than DFT orthogonal transforms as, for example, discrete cosine transform or Hadamard transforms [27, 43]. Furthermore, the RDFT can be applied to signal subintervals that can be overlapping or not [25]. In this case, data processing becomes similar to the methods used in discrete cosine transform-based denoising [44]. Our future work will concentrate on applying the adaptive robust estimators for processing signals embedded in non-Gaussian noise using other than DFT orthogonal transforms. 


\section{Acknowledgment}

The work of Igor Djurović is supported in part by the Ministry of Science and Education of Montenegro.

\section{References}

[1] H. L. Van Trees, Detection, Estimation and Modulation Theory, McGraw-Hill, New York, NY, USA, 1966.

[2] A. D. Spaulding and D. Middleton, "Optimum reception in an impulsive interference environment-part I: coherent detection," IEEE Transactions on Communications, vol. 25, no. 9, pp. 910-923, 1977.

[3] C. L. Nikias and M. Shao, Signal Processing with Alpha-Stable Distributions and Applications, John Wiley \& Sons, New York, NY, USA, 1995.

[4] S. I. Resnick, "Heavy tail modeling and teletraffic data," Annals of Statistics, vol. 25, no. 5, pp. 1805-1849, 1997.

[5] J. P. Huber, Robust Statistics, John Wiley \& Sons, New York, NY, USA, 1981

[6] J. G. Gonzalez, Robust techniques for wireless communications in non-Gaussian environments, Ph.D. thesis, University of Delaware, Newark, Del, USA, 1997.

[7] J. Astola and P. Kuosmanen, Fundamentals of Nonlinear Digital Filtering, CRC Press, Boca Raton, Fla, USA, 1997.

[8] A. V. Oppemheim and R. W. Schafer, Digital Signal Processing, Prentice Hall, Englewood Cliffs, NJ, USA, 1975.

[9] G. R. Arce, Nonlinear Signal Processing: A Statistical Approach, John Wiley \& Sons, New York, NY, USA, 2005.

[10] J. G. Gonzalez and G. R. Arce, "Statistically-efficient filtering in impulsive environments: weighted myriad filters," EURASIP Journal on Applied Signal Processing, vol. 2002, no. 1, pp. 4-20, 2002.

[11] S. Kalluri and G. R. Arce, "Adaptive weighted myriad filter algorithms for robust signal processing in $\alpha$ a-stable noise environments," IEEE Transactions on Signal Processing, vol. 46, no. 2, pp. 322-334, 1998.

[12] I. Shmulevich and G. R. Arce, "Spectral design of weighted median filters admitting negative weights," IEEE Signal Processing Letters, vol. 8, no. 12, pp. 313-316, 2001.

[13] V. Katkovnik, "Robust M-periodogram," IEEE Transactions on Signal Processing, vol. 46, no. 11, pp. 3104-3109, 1998.

[14] I. Djurović, V. Katkovnik, and L. Stanković, "Median filter based realizations of the robust time-frequency distributions," Signal Processing, vol. 81, no. 8, pp. 1771-1776, 2001.

[15] I. Djurović, L. Stanković, and J. F. Böhme, "Robust Lestimation based forms of signal transforms and timefrequency representations," IEEE Transactions on Signal Processing, vol. 51, no. 7, pp. 1753-1761, 2003.

[16] I. Djurović, L. J. Stanković, and J. F. Böhme, "Myriad filter based form of the DFT," in Proceedings of the 11th European Signal Processing Conference (EUSIPCO '02), pp. 433-436, Toulouse, France, 2002.

[17] I. Djurović, V. Lukin, and A. Roenko, "Removal of $\alpha$-stable noise in frequency modulated signals using robust DFT forms," Telecommunications and Radioengineering, vol. 61, no. 7, pp. 574-590, 2004.

[18] I. Djurović and V. V. Lukin, "Robust DFT with high breakdown point for complex-valued impulse noise environment," IEEE Signal Processing Letters, vol. 13, no. 1, pp. 25-28, 2006.

[19] A. Roenko, V. Lukin, I. Djurović, A. Kurekin, and A. Zelensky, "Filtering of frequency modulated signals embedded in $\alpha$ stable noise using robust DFT forms," in Proceedings of the
International Conference on Modern Problems of Radio Engineering, Telecommunications and Computer Science (TCSET '06), pp. 228-231, Lviv-Slavsko, Ukraine, 2006.

[20] V. V. Lukin, A. A. Roenko, I. Djurović, and L. J. Stanković, "Robust DFT based on adaptive censored estimate for FM signal processing in non-Gaussian noise environment," in Proceedings of the 9th International Symposium on Signal Processing and Its Applications (ISSPA '07), pp. 1-4, Sharjah, United Arab Emirates, 2007.

[21] I. Djurović and L. Stanković, "An algorithm for the Wigner distribution based instantaneous frequency estimation in a high noise environment," Signal Processing, vol. 84, no. 3, pp. 631-643, 2004.

[22] I. Djurović, L. Stanković, and B. Barkat, "Robust timefrequency distributions based on the robust short time Fourier transform," Annales des Télécommunications, vol. 60, no. 5-6, pp. 681-697, 2005.

[23] I. Djurović, "Time-frequency analysis for SAR and ISAR imaging," in Geospatial Visual Analysis, Part 2, pp. 113-127, Springer, Amsterdam, The Netherlands, 2009.

[24] A. Kurekin, D. Marshall, D. Radford, K. Lever, and V. Lukin, "Robust processing of SAR hologram data to mitigate impulse noise impairments," in Proceedings of the 8th International Conference on Information Fusion (FUSION '05), p. 8, Philadelphia, Pa, USA, July 2005.

[25] I. Djurović and V. V. Lukin, "Robust DFT-based filtering of pulse-like FM signals corrupted by impulsive noise," Signal, Image and Video Processing, vol. 1, no. 1, pp. 39-51, 2007.

[26] I. Djurović and V. V. Lukin, "Estimation of single-tone signal frequency by using the L-DFT," Signal Processing, vol. 87, no. 6, pp. 1537-1544, 2007.

[27] I. Djurović, L. Stanković, and J. F. Böhme, "Robust Lestimation based forms of signal transforms and timefrequency representations," IEEE Transactions on Signal Processing, vol. 51, no. 7, pp. 1753-1761, 2003.

[28] J. T. Astola, K. O. Egiazarian, G. I. Khlopov et al., "Application of bispectrum estimation for time-frequency analysis of ground surveillance Doppler radar echo signals," IEEE Transactions on Instrumentation and Measurement, vol. 57, no. 9, pp. 1949-1957, 2008.

[29] R. Suoranta, Amplitude domain approach to digital filtering. Theory and applications of L-filters, Doctor of Technology thesis, Technical Research Centre of Finland, Espoo, Finland, 1995.

[30] V. V. Lukin, V. P. Melnik, A. B. Pogrebniak, and A. A. Zelensky, "Techniques and algorithms of speckle noise reduction for one-look SAR images," in Proceedings of the European Conference on Synthetic Aperture Radar (EUSAR '96), pp. 167-170, Konigswinter, Germany, 1996.

[31] S. K. Abramov, V. V. Lukin, A. A. Zelensky, and J. T. Astola, "Blind evaluation of noise variance in images using myriad operation," in Image Processing: Algorithms and Systems, vol. 4667 of Proceedings of SPIE, pp. 192-203, San Jose, Calif, USA, January 2002.

[32] V. V. Lukin, S. K. Abramov, A. A. Zelensky, and J. T. Astola, "Use of minimal inter-quantile distance estimation in image processing," in Mathematics of Data/Image Pattern Recognition, Compression, and Encryption with Applications IX, vol. 6315 of Proceedings of SPIE, pp. 1-12, San Diego, Calif, USA, August 2006.

[33] J. Ilow, D. Hatzinakos, and A. N. Venetsanopoulos, "Performance of FH SS radio networks with interference modeled 
as a mixture of Gaussian and alpha-stable noise," IEEE Transactions on Communications, vol. 46, no. 4, pp. 509-520, 1998.

[34] J. L. Hodges and E. L. Lehmann, "Estimates of location based on rank tests," The Annals of Mathematical Statistics, vol. 34, no. 2, pp. 598-611, 1963.

[35] A. Hoyland, "Robustness of the Wilcoxon estimate of location against a certain dependence," The Annals of Mathematical Statistics, vol. 39, no. 4, pp. 1196-2001, 1968.

[36] V. V. Lukin, S. K. Abramov, and N. O. Tulyakova, "Dynamic characteristics of some nonlinear 1-D filters," in Proceedings of the Finnish Signal Processing Symposium (FINSIG '99), pp. 184-188, Oulu, Finland, 1999.

[37] J. G. Gonzalez and G. R. Arce, "Optimality of the myriad filter in practical impulsive-noise environments," IEEE Transactions on Signal Processing, vol. 49, no. 2, pp. 438-441, 2001.

[38] A. Roenko, V. Lukin, I. Djurović, and S. Abramov, "Adaptation of sample myriad tunable parameter to characteristics of SaS distribution," in Proceedings of the 12th International Conference on Mathematical Methods in Electromagnetic Theory (MMET '08), pp. 418-420, Odessa, Ukraine, July 2008.

[39] V. Lukin, A. Roenko, S. Abramov, I. Djurović, and J. Astola, "Bootstrap based adaptation of sample myriad to characteristics of $\mathrm{S} \alpha \mathrm{S}$ distribution data," in Proceedings of the IEEE International Symposium on Circuits and Systems (ISCAS '09), pp. 1205-1208, May 2009.

[40] G. Taguchi, "Adaptive $\alpha$-trimmed mean filter with excellentdetail preserving," in Proceedings of the International Conference on Acoustics, Speech and Signal Processing (ICASSP '94), pp. 61-64, 1994.

[41] H. S. Lim, C. T. Chee, and C. H. Teik, "On the optimal alpha$\mathrm{k}$ curve of the sample myriad," IEEE Signal Processing Letters, vol. 14, no. 8, pp. 545-548, 2007.

[42] A. Roenko, I. Djurović, V. Lukin, and A. Zelensky, "Accuracy improvement of the Wigner distribution estimate in nonGaussian noise environment by means of clipping technique application," in Proceedings of the International Conference on Modern Problems of Radio Engineering, Telecommunications and Computer Science (TCSET'08), pp. 362-365, Lviv-Slavsko, Ukraine, 2008.

[43] I. Djurović and L. J. Stanković, "Robust Hadamard transform," in Proceedings of the 9th IEEE Mediterranean Conference on Control and Automation, Dubrovnik, Croatia, 2001.

[44] V. V. Lukin, D. V. Fevralev, S. K. Abramov, S. Peltonen, and J. Astola, "Adaptive DCT-based 1-D filtering of Poisson and mixed Poisson and impulsive noise," in Proceedings of the International Workshop on Local and Non-Local Approximation in Image Processing (LNLA '08), p. 8, Lausanne, Switzerland, August 2008, CD ROM. 